Archives de sciences sociales des religions

127 | juillet - septembre 2004

Max Weber, la religion et la construction du social

\title{
Georg Jellinek, Max Weber, le politique et la tâche de la sociologie des religions
}

Jean Martin Ouédraogo

\section{OpenEdition}

Édition électronique

URL : http://journals.openedition.org/assr/1056

DOI : $10.4000 /$ assr. 1056

ISSN : $1777-5825$

Éditeur

Éditions de l'EHESS

Édition imprimée

Date de publication : 1 juillet 2004

Pagination : 105-137

ISBN : 2-222-96751-1

ISSN : 0335-5985

Référence électronique

Jean Martin Ouédraogo, «Georg Jellinek, Max Weber, le politique et la tâche de la sociologie des religions », Archives de sciences sociales des religions [En ligne], 127 | juillet - septembre 2004, mis en ligne le 25 juin 2007, consulté le 19 avril 2019. URL : http://journals.openedition.org/assr/1056 ; DOI : 10.4000/assr.1056 
Arch. de Sc. soc. des Rel., 2004, 127, (juillet-septembre 2004) 105-137

Jean Martin OUÉDRAOGO

\section{GEORG JELLINEK, MAX WEBER, LE POLITIQUE ET LA TÂCHE DE LA SOCIOLOGIE DES RELIGIONS}

Sur plusieurs questions décisives, Max Weber a inscrit ses réflexions dans le sillage des travaux de Jellinek(2). Il en est de même de ce qu'il entreprendra d'élucider dans sa sociologie des religions. Mais, il ne répètera pas simplement ce dernier. Il confèrera à son entreprise, dans le domaine des religions notamment, une note personnelle, à la fois théorique et politique. Cette sociologie des religions, telle qu'elle fut explicitement pensée et mise en œuvre, avait pour objet de mettre

Pour des raisons d'ordre matériel, plusieurs séjours de recherche en Allemagne (ArbeitsstelleArchiv Max Weber, (Munich), Institut Max-Planck für Geschichte (Göttingen)) ont été nécessaires à la rédaction de ce texte. Un soutien initial de la Fondation Fritz-Thyssen, et par la suite de la Société MaxPlanck m'ont permis d'accomplir ce travail. Je remercie les professeurs Theo Stammen et Hartmut Lehmann qui m'ont soutenu dans ce projet. Au cours de la rédaction de ce travail, j'ai essayé de tirer profit des discussions menées avec messieurs : Hans-Erich Bödeker, Robert Kremer, Jürgen Schlumbohm, Jean Séguy et Rudolf Vierhaus. Qu'ils en soient ici également remerciés.

(1) Cf. Lettre adressée à Mina Tobler datée du 17 janvier 1919, citée dans Max WeBER, Zur Neuordnung Deutschlands. Schriften und Reden 1918-1920. Band 1/16 der Max Weber Gesamtausgabe, Hrsg. von Wolfgang J. Mommsen in Zusammenarbeit mit Wolfgang Schwentker, Tübingen, J.C.B. Mohr (Paul Siebeck), 1988, p. 19 note 53. Les textes de cette édition seront abrégés, par la suite, par MWG.

(2) Cet article poursuit la recherche initialement engagée sur le rapport entre religion et modernité politique chez Max Weber dans laquelle l'influence de Jellinek a été mise en lumière ; cf. Jean Martin OuÉDRAOGO, "Sociologie religieuse et modernité politique chez Max Weber », Revue Européenne des Sciences Sociales, 34, 1996, 106, pp. 25-49. Paradoxalement, peu de chercheurs se sont véritablement penchés sur les relations intellectuelles entre les deux hommes. On lira : sur l'État, Andreas ANTER "Georg Jellineks wissenschaftliche Politik: Positionen, Kontexte, Wirkungslinien », Politische Vierteljahresschrift, 39, 1998, 3, pp. 503-526; idem, Max Weber Theorie des modernen Staates. Herkunft und Bedeutung, Berlin, Dunker \& Humblot, 1995 ; Stefan Breuer, Georg Jellinek und Max Weber. Von der sozialen zur soziologischen Staatslehre, Baden-Baden, Nomos Verlag, 1999 ; Gangolf HüBINGER, « Staatstheorie und Politik als Wissenschaft im Kaiserreich : G. Jellinek, O. Hinze, M. Weber » in Hans Maier, Ulrich Matz, Kurt Sontheimer, Paul-Ludwig Weinacht, Hrsg., Politik, Philosophie, Praxis. Festschrift für W. Hennis zum 65. Geburtstag, Stuttgart, 1988, pp. 143-161. 
au jour les principes religieux qui furent à l'origine du double avènement de l'accès de la bourgeoisie au pouvoir politique et de la politique de puissance (Machtpolitik) en Angleterre. La réception de cette sociologie des religions s'est donc constituée, en fait, sur la base d'un malentendu aux implications considérables : d'abord, en réduisant cette sociologie à l'étude du rapport entre religion(s) et transformation économique (capitalisme) ; ensuite, au regard de la démarche qui sous-tend l'entreprise de Weber, elle n'a retenu, de notre point de vue, que le moment préliminaire induit par le modèle d'analyse choisi par Weber. Ce faisant, ce qui, initialement apparut à Weber comme un détour nécessaire à sa recherche, est devenu, au fil des années, chemin et destination à la fois de cette dernière.

Afin de justifier la perspective que nous venons d'esquisser, nous nous proposons dans ce texte : de signaler brièvement le problème aussi bien théorique que politique qui s'imposa à Weber en amont de sa sociologie des religions; de prendre la mesure de la signification de "l'incitation» (Max Weber) d'origine jellinekienne par rapport à ce problème ; d'examiner la tâche et la manière dont Weber essaya de s'en acquitter dans l'optique de le résoudre ; et enfin, à partir de ce qui s'est dessiné très tôt comme l'aval de ces études, de fournir quelques indications susceptibles de justifier l'hypothèse d'une articulation entre ses recherches et sa pensée politique.

Nous proposons de donner préalablement un aperçu des discussions autour des considérations qui commandèrent l'entreprise wébérienne.

\section{Motif et intention de Max Weber dans la mise en ouvre de la sociologie des religions}

Quel motif et quelle intention Weber avait-il en s'engageant dans son étude sur l'Éthique protestante et l'esprit du capitalisme et par la suite dans l'ensemble de ses réflexions qui constitue sa sociologie des religions? D'un point de vue chronologique, ces textes ont été écrits, certes à des moments différents, mais ils manifestent une certaine continuité. Cette continuité, d'ailleurs affirmée par Weber, est de deux ordres: elle est d'ordre problématique et d'ordre thématique à la fois (3). L'orientation théorique générale imprimée à ces textes a justifié, aux yeux

(3) Sur cette continuité, on se reportera ici à Max WEBER, « La morale économique des grandes religions ", Archives de Sociologie des Religions, 5, 1960, p. 7, cf. note. De même à la lettre qu'il adressa le 22 juin 1915 à son éditeur P. Siebeck que cite Wolfgang Schluchter; cf. Wolfgang SCHLUCHTER, Religion und Lebensführung, Bd. 2, Francfort a.M., 1988, p. 609. Il s'agit ici, d'abord, des textes regroupés en 1919 sous le titre Die Wirtschaftsethik der Weltreligionen (Éthique économique des religions mondiales) ; cf. Max WeBER, Die Wirtschaftsethik der Weltreligionen, 3 vol., Tübingen, J.C.B. Mohr (Paul Siebeck), 1986. Ensuite du chapitre V, intitulé « Sociologie religieuse (les types de communalisation religieuse) », texte posthume inclus par ses différents éditeurs dans Max WEBER, Économie et Société, vol. 1, Paris, Plon, 1971, pp. 429-632. Par la suite en abrégé, ES. Sur la discussion relative au rapport entre ces textes, on se refèrera à Wolfgang SCHLuchter, Religion und Lebensführung, vol. 2, op. cit., pp. 557-596. Johannes Winckelmann, Max Webers hinterlassenes Hauptwerk, Tübingen, J.C.B. Mohr (Paul Siebeck), 1986, p. 42. 
de B. Nelson et de L. Dumont, de les considérer comme une contribution importante à l'étude comparative des civilisations (4).

Du point de vue de l'objet de ses recherches, Weber se serait principalement intéressé aux rapports entre "religions et économie », c'est-à-dire aux conditions religieuses du développement du capitalisme moderne. Telle est l'image encore dominante dans la littérature secondaire, correspondant à la place qui lui est attribuée en tant que fondateur dans l'histoire de la sociologie moderne. Cependant, un sentiment de perplexité naît, dès lors que l'on s'interroge sur le motif et l'intention qui ont pu conduire Weber à s'intéresser de manière aussi intense aux conditions religieuses du développement de l'économie moderne. En effet, il faut rappeler qu'en 1904, au moment où Weber s'engagea dans cette recherche sur "l'Ethique Protestante", l'Allemagne était déjà devenue un pays hautement industrialisé. Entre 1870 et 1904, ce pays est passé, de manière décisive, d'une économie dominée par l'agriculture et la petite entreprise à une économie marquée par la prédominance de la grande industrie. Personnellement, Weber n'hésite pas à utiliser l'expression de "capitalisme développé" (Hochkapitalismus) pour désigner cette situation économique ou même à considérer l'avancée du capitalisme comme un phénomène désormais universel et une réalité devenue incontournable (5). Pourquoi donc, dans un tel contexte, s'intéresser avec une telle abnégation à une question qui n'est pas inscrite à l'ordre du jour des questions économiques ou encore à des faits qui sont, du point de vue historique, en décalage par rapport à la situation réelle de l'Allemagne?

Notre perplexité s'accroît à mesure que l'on prend également en considération le fait que Weber n'est pas le premier à introduire le thème "protestantisme et capitalisme" dans la littérature scientifique. Paul Münch et Thomas Nipperdey ont récemment reconstitué l'histoire de ce thème avant l'entrée en scène de Weber (6). Sur ce point, Weber avait d'ailleurs bien laissé entendre : "affirmer cette relation ne constitue donc pas une "nouveauté"...». Et il poursuit "Laveleye, Matthew Arnold et d'autres en ont déjà traité (7) ». S'agissait-il alors pour lui de fournir un fondement théorique plus assuré aux travaux antérieurs portant sur le rapport entre protestantisme et capitalisme ? Puisque, écrit-il aussi, il s'agit «d'abandonner les représentations vagues et générales » qui ont prévalu dans la recherche historique (8). Tout lecteur de son texte est en mesure de constater qu'il ne s'agit pas en

(4) Cf. Benjamin Nelson, « Max Weber's “Author's introduction” (1920): A Master Clue to his Main Aims », Sociological Inquiry, 44, 1974, pp. 269-278 ; idem, « Weber, Troeltsch, Jellinek as comparative historical sociologists ", Sociological Analysis, 36, 1975, 3, pp. 229-240. Louis Dumont, Essais sur l'individualisme, une perspective anthropologique sur l'idéologie moderne, Paris, Seuil, 1983.

(5) Max Weber, " Geleitwort », Archiv für Sozialwissenschaft und Sozialpolitik, 19, 1904, pp. II, IV et V. Pour une vue générale des grandes transformations économiques, sociales, scientifiques et techniques, on se réfèrera ici en particulier à Hans-Ulrich WeHLER, Das Deutsche Kaiserreich 1871-1918, Göttingen, Vandenhoeck \& Ruprecht, 1977, pp. 19-59.

(6) Cf. Paul MüNICH « The Thesis before Weber: an Archeology », in Hartmut Lehmann, Guenther Roth, eds., Weber's Protestant Ethic. Origins, Evidence, Contexts, Washington D.C., Cambridge University Press, 1993, pp. 51-72. Thomas NiPPERdey, « Max Weber, Protestantism and the Debate around 1900 », in Hartmut Lehmann, Guenther Roth, eds., op. cit., pp. 73-81.

(7) Max Weber, l'Éthique protestante et l'Esprit du capitalisme, Paris, Plon, 1967, p. 42, note 22. Par la suite en abrégé É.P. Voir également, ÉP, p. 237, note 84.

(8) ÉP, p. 44. 
l'occurrence d'un texte de vulgarisation des travaux antérieurs auxquels il aurait fourni simplement une base théorique jugée indispensable. Quelles sont donc les considérations qui ont pu être à l'origine de cette sociologie des religions ?

Après plusieurs décennies de recherches ininterrompues sur les travaux de Weber, ces questions sont encore sans réponse décisive quoiqu'elles aient accompagné l'histoire de la réception de ses textes. Selon Gianfranco Poggi, « [...] nous savons peu de choses au sujet de la genèse de l'Éthique protestante [...] (9) ». Cet avis est partagé par Hartmut Lehmann et Wilhelm Hennis. En introduction à un texte sur la réception de la thèse de Weber, le premier déclare : «L'on doit insister au préalable sur le fait que l'on sait extraordinairement peu de choses sur l'arrièreplan intellectuel et personnel de la célèbre étude de Weber, et que, ce que nous savons, n'est pas exempt d'absurdités et de contradictions». Selon le second «les considérations qui furent déterminantes pour Max Weber ne sont toujours pas éclaircies (10). » En fait, au sujet de l'intention qui a pu conduire Weber à s'attaquer en particulier à la question du rapport entre l'Éthique protestante et l'esprit du capitalisme plusieurs hypothèses ont été avancées, ne serait-ce que pour comprendre l'origine de ce texte en particulier. On pourrait ici, par souci de commodité, les regrouper selon trois modèles, étant entendu que les mêmes auteurs proposent souvent plusieurs hypothèses à la fois (11) :

- l'hypothèse d'ordre psychologique : avec un accent plus psychanalytique, cette explication a été proposée par A. Mitzman. L'interprétation historique qu'annonce le titre de son livre est en réalité secondaire (12). Weber aurait été confronté à la culture répressive et autoritaire de l'Allemagne wilhelminienne que symbolisa son père. De ce point de vue, l'Éthique protestante serait un "acte d'auto-libération » (13). Dans cette approche, la compréhension de «l'œuvre » est déduite de la compréhension de «l'homme». Les intentions scientifiques et le choix des problèmes sont ramenés à la biographie de l'auteur, et en dernière analyse aux rapports que Weber entretint avec son père (représentant du «patriarcat» et de "l'ordre libéral ») et avec sa mère (représentante de la rigueur morale calviniste, puisque d'origine huguenotte). Ce type d'herméneutique produit trois conséquences majeures : l'unité de l'œuvre n'est pas recherchée dans l'œuvre elle-même, mais dans celle préalablement reconstruite de la personnalité de son auteur. Les thèmes de l'œuvre sont définis à partir d'un critère de pertinence non-interne aux textes. Non pas un critère,

(9) Gianfranco Poggi, Calvinism and the Capitalist Spirit. Max Weber's Protestant Ethic, Londres, Mc Millan, 1983, p. 5.

(10) Hartmut LehmanN, «Asketischer Protestantismus und ökonomischer Rationalismus. Die Weber-These nach zwei Generationen ", in Hartmut Lehmann, Max Webers Protestantische Ethik, Göttingen, Kleine Vandenhoeck-Reihe, 1996, p. 9. Wilhelm HenNIs, Max Webers Wissenschaft vom Menschen, Tübingen, J.C.B. Mohr (Paul Siebeck), 1996, p. 312. Hennis soutient néanmoins une autre idée. En effet, "l'éthique protestante, mais aussi l'éthique économique des religions mondiales [ont été écrits] de manière purement arbitraire ; aucune contrainte extérieure et surtout aucune contrainte d'un calendrier [de travail] ne le pressaient »cf. ibid., p. 207. l'italique est de nous.

(11) Il ne s'agit pas pour nous de classer ici l'ensemble de ceux qui ont proposé une explication, mais de signaler simplement l'absence de consensus, en évoquant brièvement l'éventail des hypothèses proposées.

(12) Arthur Mitzman, The Iron Cage. An Historical Interpretation of Max Weber, New Brunswick, Transaction Books, 1985.

(13) Ibid., p. 175. 
interne, d'ordre logique ou théorique, mais un critère plutôt externe, relatif au passé affectif de l'auteur. Enfin, les rapports internes entre les textes, les thèmes, les arguments et leurs développements disparaissent au profit d'un principe de corrélation externe. Ainsi, pour nous en tenir à la sociologie des religions, l'investigation de "l'ascétisme intra-mondain » (puritanisme), devait avoir pour Weber un intérêt plus qu'académique (14).

Mitzman croit avoir décelé cet intérêt à la fin de l'Éthique protestante où Weber s'interroge sur l'actualité du rapport entre puritanisme et capitalisme. Dans le passage en question, Weber nous dit que ce rapport n'est plus actuel et que le capitalisme est devenu autonome (15). Les intérêts matériels auxquels les puritains accordèrent peu d'importance - " "un 'léger manteau' que l'on pouvait à tout moment jeter" "- s'est mué, grâce au destin, en " une carapace dure comme de l'acier » (16). D'après Mitzman, cet « habitat dur comme de l'acier» rappelle la «maison sécurisante de la génération» de son père. Aux yeux de Mitzman, le développement historique esquissé par Weber, l'ordre d'exposition qu'il choisit pour rendre compte de ce développement historique, entretiendraient une analogie avec son propre développement personnel. De ce point de vue, l'éthique protestante constitue un chapitre de la "propre généalogie morale de Weber ». L'intérêt de Weber dans cette investigation résiderait donc dans cet auto-dévoilement au lecteur de sa propre évolution psychologique (17): une simple hypothèse non étayée et non étayable, croyons-nous. La suite de la sociologie des religions serait thématiquement structurée autour de l'opposition entre " ascétisme » et " mysticisme ». Là aussi, Weber aurait poursuivi son auto-dévoilement. Il se serait tourné vers une quête intérieure de type mystique, une «alternative » à la "rationalisation » puritaine d'avant l'érection de la "cage d'acier» (18). Il est vrai que la recherche comparative entre les cultures religieuses a pris de l'ampleur chez Weber quelques années plus tard. Mais ce comparatisme existait déjà dans l'Éthique protestante. L'opposition entre ascétisme et contemplation mystique n'est ni la seule ni même centrale dans ce travail comparatif. Au-delà de ce privilège arbitrairement accordé à cette dichotomie, l'ouvrage de Mitzman repose sur deux prémisses erronées : premièrement, l'assimilation de l'avènement de l'unité allemande de 1871 avec l'instauration d'un ordre politique libéral (voir son introduction); et deuxièmement, l'interprétation en terme de «décadence» qu'aurait faite Weber des convictions libérales et socialistes de son temps (19).

(14) Ibid., p. 172.

(15) Cf. $E P$, pp. 250-251.

(16) Ibid. (trad. modifiée). Les traductions françaises et américaines de «Gehäuse » par cage d'acier introduit une nuance que nous ne trouvons pas dans le mot allemand. Weber n'emploie pas le mot «Käfig ». Mitzman a eu bien conscience de cette nuance et préféra traduire par "a housing hard as steel ». Mais il reprend néanmoins la traduction de "Gehäuse » "iron cage » de Talcott Parsons dans le titre de son livre et aussi dans l'esprit de son interprétation. Cf. Idem., The Protestant Ethic and the Spirit of Capitalism, trans. by Talcott Parsons, New York, 1948, p. 181.

(17) Cf. Arthur Mitzman, op. cit. p. 175.

(18) Ibid., pp. 194 et 200.

(19) Ibid., p. 176. 
L'explication psychologique a été récemment suggérée aussi par Hartmut Lehmann: selon cette explication, l'ouvrage de Weber relèverait d'une sorte "d'auto-affirmation scientifique ». Grâce à ce texte, Weber aurait cherché - et réussi - à s'affirmer dans le champ universitaire après sa retraite forcée en 1903, occasionnée par les dépressions qu'il a connues dès 1898 et la longue interruption de sa vie académique qui en a suivi (20). Lehmann recourt ainsi à l'expérience existentielle de Weber pour expliciter certains passages centraux de l'Éthique protestante. Donnons ici quelques exemples : l'esseulement qu'a connu Weber au cours de sa maladie a été une condition décisive de la capacité dont il a fait preuve dans la description de "l'esseulement du calviniste" confronté aux conséquences de la théorie de la prédestination. De même, le thème de "l'élection" et de la "grâce" serait à mettre en rapport avec la conception que développa Weber au sujet du sens de l'engagement au service de la science qu'il se voyait personnellement mettre en œuvre. Bref, à lire le texte de Lehmann, on est frappé par la pertinence des correspondances entre faits d'origine biographique et contenu du texte scientifique. La démonstration de Lehmann ne laisse pas de doute sur le fait que la force argumentative de la thèse de Weber ait été métaphoriquement, dirons-nous, nourrie par son propre vécu. Cependant, le principal problème sur lequel bute ce modèle explicatif de même que celui de Mitzmann, est la difficulté dans laquelle on se trouve, quand on songe à l'extension de sa validité à l'ensemble des textes de Weber relevant de la sociologie des religions et entretenant avec "l'Éthique protestante" des rapports de nature thématique et programmatique. De notre point de vue, la validité d'une hypothèse de lecture doit pouvoir être revendiquée pour l'ensemble des textes relevant du même registre. Dans la mesure où il s'agit de clarifier le motif et l'intention de Weber, sa recevabilité doit l'être relativement à ces conditions. C'est-à-dire, au regard des thème(s), du programme et de la perspective théorique qu'il déploie dans ces textes. Évidemment ces questions n'annulent pas l'importance du facteur biographique dans la genèse des textes scientifiques.

- L'approche intellectualiste: plusieurs auteurs considèrent “l'Éthique protestante" comme un texte ayant été élaboré pour réfuter un auteur ou des auteurs précis, ou un courant intellectuel. Déjà pour Georg Lukacs, ce texte représente " une forme "raffinée" de critique du matérialisme historique » et aurait eu pour fonction de "dépasser» le marxisme (21). Ce texte de Weber a aussi été vu comme une réfutation de la thèse de Sombart sur le rapport des juifs au capitalisme (22). Enfin

(20) Hartmut Lehmann, Max Webers »Protestantische Ethik«, op. cit., pp. 109-127.

(21) Georg Lukacs, Die Zerstörung der Vernunft, Berlin, Aufbau-Verlag, 1955, p. 477. Cette idée a été reprise par différents auteurs : ainsi par Wolfgang J. Mommsen : « en réalité, d'importantes parties de son œuvre l'éthique protestante en particulier, se laissent interpréter comme une discussion avec Marx "; cf. Wolfgang J. Mommsen, Max Weber. Gesellschaft, Politik und Geschichte, Frankfurt a. M., Suhrkamp, 1982, p. 101. Également chez Wolfgang Schluchter, Religion und Lebensführung, Bd. 1, op. cit. Cette tradition d'interprétation prend évidemment en considération les propres propos de Weber au sujet de son rapport à Marx; il s'est exprimé en maint endroit, notamment dans «l'Éthique Protestante ». Dans ce texte n'a-t-il pas en effet laissé entendre qu'il s'agissait pour lui d'une critique positive du matérialisme historique ? cf. ÉP, pp. 53 et $s q$., p. 79. Pour une critique de cette perspective, voir Steven SEIDMAn, Liberalism and the Origins of European Social Theory, Berkeley, University of California Press, 1983, cf. en particulier, pp. ix-x.

(22) Il s'agit de Werner Sombart, Die Genesis des Kapitalismus, Bd. 1. Die Theorie der kapitalistischen Entwicklung, Bd. 2, Leipzig, 1902. La question a été de nouveau examinée par Lehmann ; cf. Hartmut LeHmanN, op. cit., pp. 94-108. 
récemment, Hans G. Kippenberg a suggéré que le livre de Weber participerait " d'une protestation contre les idées courantes de progrès (23). » Il est évident que Weber s'est situé par rapport à ces auteurs et à bien d'autres dans son œuvre. Il ne pouvait en être autrement. Tout travail scientifique est pris dans les exigences et les contraintes de la communication propres à ce champ. L'auto-clarification de ses propres positions par rapport aux courants alternatifs est de l'ordre de ces contraintes. Tel a été le cas aussi de la sociologie des religions de Weber, en ce qui concerne notamment sa position vis-à-vis de certains intellectuels de son époque. De même que l'évocation des idées dont s'est nourri un auteur, la référence aux idées auxquelles il s'est opposé, ne peut constituer une solution au problème fondamental du motif et de l'intention qui sont à l'origine d'une œuvre.

- Une dernière hypothèse reste à examiner. Elle est de G. Roth. Bien qu'elle pèche par quelques-unes de ses implications, elle a le mérite d'offrir à la recherche une voie qui vaut la peine d'être envisagée. Selon Roth, « une frustration politique poussa Weber vers l'histoire religieuse comparative et la sociologie (24). " Le motif de la sociologie des religions de Weber est à rechercher du côté de ses attentes ou de ses convictions politiques : attentes non satisfaites ou convictions contrariées. Roth va plus loin. À ses yeux, l'Éthique protestante serait un «wishful political thinking (25). " Son hypothèse relative au motif qui se trouve à l'origine du livre va de pair avec son appréciation du contenu de celui-ci. Weber aurait sublimé dans son ouvrage un manque réel de nature politique. Plusieurs objections peuvent être émises à l'encontre de cette grille de lecture. S'il faut prendre au sérieux les préoccupations politiques de Weber qui se trouvent en amont de cette sociologie, faudrait-il aussi voir dans cette sociologie une idéologie simplement drapée d'oripeaux scientifiques, une sorte d'accommodement de cette idéologie à l'atmosphère positiviste de l'époque ? Ce serait aller vite en besogne. À s'en tenir à l'Éthique protestante, si l'on présuppose ici la lecture que fait Roth de cette sociologie, le moins que l'on puisse dire est que cette idéologie serait anachronique et nostalgique. Weber aurait-il été personnellement insensible à son anachronisme en proposant des idées qu'il considère explicitement comme n'étant plus nécessaires au type de capitalisme de son temps ? Il nous laisse en effet comprendre à la fin de son texte sur ascétisme et esprit capitaliste, que le capitalisme n'a plus besoin du soutien spirituel de l'ascétisme protestant, dans la mesure où il repose désormais sur « une base mécanique (26). » Dans ces conditions, faut-il néanmoins voir dans son entreprise, une opération déguisée de mobilisation politique à partir de la simple exhumation des références religieuses qui ont été favorables au développement du capitalisme dans sa phase hérö̈que ?

S'il n'existe pas d'enjeu scientifique véritable derrière la revendication de la neutralité axiologique, de la recherche de l'objectivité dans l'étude des faits

(23) Hans G. KipPenberg, À la découverte de l'histoire des religions. Les sciences religieuses et la modernité, Paris, Éditions Salvator, 1999, p. 223.

(24) Guenther Rотн, «Zur Entstehung und Wirkungsgeschichte von Webers "Protestantische Ethik" ", in Bertram Schefhold, Guenther Rotн et al., eds., Vademekum zu einem Klassiker der Geschichte der ökonomischen Rationalität, Düsseldorf, Verlag Wirtschaft und Finanzen, 1992, p. 207.

(25) Guenther Rотн, «Weber the Would-Be Englishman: Anglophilia and Family History » in Hartmut Lehmann, Guenther Roth, eds., Weber's Protestant Ethic, op. cit., p. 121.

(26) ÉP, p. 250. 
religieux, pourquoi Weber aurait-il abandonné la perspective esquissée dans sa leçon inaugurale de Fribourg - qui avait au moins le mérite d'être claire quant à son dessein - pour travestir de la sorte ses préoccupations politiques en s'investissant dans l'étude du rapport entre protestantisme et capitalisme et d'une manière générale dans la sociologie des religions ? Envisager la sociologie des religions de Weber comme un gigantesque travestissement politique pose évidemment problème. La revendication et l'effort intellectuel fournis par Weber pour attribuer un statut scientifique à ses textes perdent ici toute crédibilité (27). L'objection décisive à la perspective de Roth serait la suivante : avec l'Éthique Protestante, Max Weber abandonne le champ de l'économie politique pour investir celui de la sociologie des religions. Pourquoi n'a-t-il pas simplement poursuivi ses travaux dans le cadre de l'économie politique ? À moins de soutenir que la "frustration politique» a été aussi pour Weber une frustration scientifique, il est difficile de comprendre son passage de l'économie politique à la sociologie des religions. Roth n'ira pas jusqu'à dire qu'il s'est agi aussi d'une frustration scientifique. Par ailleurs, Weber n'a pas complètement abandonné l'économie politique. Il a continué à la pratiquer parallèlement à sa sociologie des religions comme l'a soutenu récemment encore $\mathrm{H}$. Bruhns (28). Roth ne défendrait-il pas implicitement par hasard la thèse selon laquelle le simple fait de s'occuper de religion s'apparenterait à la production d'un opium, appliquant ainsi la célèbre thèse de Marx à Max Weber ?

Nous sommes ainsi conduit à envisager une nouvelle hypothèse. Nous la formulerons de la manière suivante : la sociologie des religions est apparue à Weber comme étant le moyen adéquat susceptible de lui permettre de maîtriser l'expérience politique. Cette expérience est aussi bien politique qu'historique. Ainsi, son passage de l'économie politique à la sociologie des religions a été essentiellement commandé par la nécessité d'une prise en charge de cette expérience. C'est en répondant aux exigences intellectuelles de celle-ci qu'il en est venu à changer de cadre intellectuel. La question de la maîtrise de cette expérience, comme il l'appréhende, n'est pas concomitante au développement de cette

(27) Il est évident que la distinction épistémologique entre science et politique perd ici toute pertinence. Cette approche de Roth nous semble correspondre à une modification de sa position initiale qui était plutôt favorable à une distinction entre ces deux sphères. Cf. Guenther RoTH, " Political Critiques of Max Weber: Some Implications for Political Sociology », American Sociological Review, 30, 1965, pp. 213 sq. Il rejoint ainsi à la fois Marianne Weber et Wolfgang J. Mommsen. Marianne Weber voyait dans la sociologie des religions une sorte d'activité « contemplative » de Weber, (un genre de fuite hors du monde) à la différence de l'activité politique ; cf. Marianne WeBer, Max Weber. Ein Lebensbild, mit einem Essay von Guenther Roth, München, Piper, 1989, pp. 173-176. Mommsen de son côté en est venu à considérer comme "naïf », " la séparation entre science et politique », parce que les présupposés de l'œuvre sociologique de Weber renverraient à une attitude fondamentale à la fois « spécifiquement intellectuelle et sociale » laquelle aurait une «dimension politique ». Nous avons du mal à voir dans cette thèse autre chose que celle défendue jadis par Christoph Steding, selon laquelle politique et science seraient identiques chez Max Weber. Selon toute vraissemblance, Mommsen considère l'entreprise scientifique de Weber comme un échec. Puisqu'il affirme par ailleurs, que «l'œuvre scientifique de [Weber], sa poursuite inflexible de la droiture intellectuelle et de l'objectivité scientifique, peut être interprétée en un certain sens comme une grandiose tentative permanente de prendre chaque fois une distance et de se créer une liberté intérieure vis-à-vis des événements politiques " ; cf. Wolfgang J. MommSEN, Max Weber und die Deutsche Politik 1890-1920, 2. Aufl.,Tübingen, J.C.B. Mohr (Paul Siebeck), 1974, p. 1 et note 10, p. 446 ; cf. Christoph Steding, Politik und Wissenschaft bei Max Weber, Breslau, Korn, 1932, pp. $11,23,56$.

(28) Cf. Hinnerk Bruhns, «Max Weber, l'économie et 1'histoire », Annales ESC, 51, 1996, 6, pp. $1259-1287$. 
sociologie. Elle n'a pas cessé de le préoccuper au cours de ses réflexions antérieures. Elle apparaît en effet comme problème dans ses travaux sur les ouvriers agricoles de l'Est de l'Elbe. Elle continue de le tarauder à l'occasion de sa leçon inaugurale de Fribourg. De ce point de vue, notre hypothèse présuppose donc une continuité entre les deux phases de la biographie intellectuelle de Weber. À cet égard, il n'existe pas de rupture entre la leçon inaugurale et l'Éthique protestante, même si, comme le reconnaît G. Roth, «Weber [...] ne relia pas explicitement son essai [l'Éthique protestante] à sa conférence de l'université de Fribourg [...] (29)».

À notre avis, il faudrait prendre au sérieux le sens que Weber a lui-même donné à son entreprise sociologique et chercher à en tirer les conséquences. Modestement, apporter une contribution à la clarification de la manière dont les " "idées" deviennent efficaces dans l'histoire (30). " Sous cette formulation, Max Weber se dote d'une grille de lecture de l'expérience politique que l'on ne peut comprendre qu'en effectuant un retour, ne serait-ce que brièvement, à ses travaux d'avant l'élaboration de l'Éthique Protestante.

Les préoccupations politiques et théoriques de Max Weber apparaissent au moment de son engagement dans les enquêtes commanditées par l'association pour la politique sociale (Verein für Sozialpolitik) (VfS) (1890), et par le congrès évangélique et social (ESK) (1892) (31). Ces deux ordres de préoccupation le conduiront à centrer sa réflexion sur l'expérience politique.

Dans le cadre de ces enquêtes, la contribution de Weber comporte un double aspect, scientifique et politique. À sa participation au niveau de l'élaboration du projet, du dépouillement et de l'interprétation des résultats, s'est greffé un apport politique correspondant à l'esprit des commanditaires des deux enquêtes. Cependant, cet apport ne s'est pas limité à ce qui aurait pu s'inscrire dans l'agenda politique des deux associations. Weber va plus loin. Il ne propose pas simplement des mesures politiques que le VfS ou l'ESK pouvaient assumer comme

(29) Guenther Roth, «The Would-Be Englishman: Anglophilia and Family History », op. cit., p. 92. [...] ajouté par nous. On notera que la conférence que prononça Weber en 1904 à Saint-Louis reprend aussi bien les résultats de ses travaux sur la situation économique et politique à l'Est de l'Elbe que certaines réflexions relatives au catholicisme, au luthéranisme et au puritanisme contenues dans l'Éthique protestante. Si bien que ce texte nous semble se situer, non pas chronologiquement mais thématiquement à l'intersection de l'économie politique et de la sociologie des religions. Cf. Max WeBER, "The Relation of the Rural Community to other Branches of Social Sciences ", in Wirtschaft, Staat und Sozialpolitik. Schriften und Reden 1900-1912. Band MWG, I/8 der MWG, ed. von Wolfgang Schluchter in Zusammenarbeit mit Peter Kurth und Birgitt Morgenbrod, Tübingen, J.C.B. Mohr (Paul Siebeck), 1998, pp. 212-243.

(30) ÉP, p. 106 ; (traduction modifiée).

(31) Encore que, comme l'a suggéré Wolfgang J. Mommsen, des allusions de nature politique apparaissaient déjà dans ses travaux antérieurs, notamment dans celui consacré au déclin de la culture antique (Untergang der antiken Kultur). Cf. Wolfgang J. Mommsen, Max Weber und die deutsche Politik, op. cit., p. 23. Voir aussi Keith TriBE, « Prussian Agriculture - German Politics: Max Weber 1892-1897 », Economy and Society, 12, 1983, 2, pp. 181-226. Sur l'histoire de la genèse des deux enquêtes, de leur mise en œuvre, du contexte politique général, et de l'implication de Weber, voir l'introduction aux textes de Weber. Cf. Max Weber, Die Lage der Landarbeiter im ostelbischen Deutschland 1892, Band I/3 der MWG, Hrsg. von Martin Riesebrodt, vol. 1, 84, pp. 1-17 ; et aussi idem, Landarbeiterfrage, Nationalstaat und Volkswirtschaftspolitik. Schriften und Reden 1892-1899, Band I/4 der MWG, Hrsg. von Wolfgang J. Mommsen in Zusammenarbeit mit Rita Aldenhoff, vol. 1, Tübingen, J.C.B. Mohr (Paul Siebeck), 1993, pp. 1-68. 
revendications vis-à-vis de l'État. Il joint à la formulation de ces mesures, une réflexion sur l'ordre politique de l'Allemagne post-bismarkienne qu'il envisage résolument en terme de transformation.

Du point de vue scientifique, les enquêtes révèlent à Weber, une « mutation dans l'organisation de la propriété terrienne dans cette région » de l'Est de l'Allemagne. Cette structure «patriarcale » se trouve désormais soumise aux effets conjugués de plusieurs facteurs socio-économiques (la concurrence internationale, la mécanisation et l'intensification de la production, l'immigration slave, la soif de liberté des ouvriers, etc.). Sa conclusion est sans appel: la forme d'organisation de la propriété terrienne historiquement connue dans cette région est condamnée à disparaître ou à se transformer en organisation capitaliste. Weber découvre aussi que cette structure ne possédait pas uniquement une fonction économique ; mais qu'elle avait aussi une fonction politique. Cette structure était à ses yeux le reflet et le fondement de l'État prussien. Elle participait à la reproduction de l'État. Parce que frappée désormais d'obsolescence sur le plan économique, l'organisation de la propriété terrienne est destinée à ne plus pouvoir jouer son rôle dans la reproduction de l'ordre politique.

Sur la base de ces considérations, quelles mesures l'État serait à même de prendre de manière à préserver ses intérêts dans cette région? Weber préconise trois mesures : offrir la possibilité aux ouvriers agricoles d'acquérir des terres, leur accorder le droit syndical, et enfin fermer les frontières à l'immigration. En formulant ces mesures, Weber se situait dans l'optique réformiste des deux associations. Cependant, après avoir constaté que les transformations à l'Est étaient symptomatiques de bouleversements profonds de l'ensemble de la société allemande, Weber tira d'autres conséquences politiques : 1) La base sociale du système politique, les grands propriétaires de domaines, est en déclin. Corrélativement, le centre de gravité de l'intelligence politique s'est déplacé de la campagne vers les villes; 2) La réalisation de l'unité allemande n'est pas «la fin de l'histoire allemande ». L'Allemagne aspire désormais à devenir « une grande puissance »; 3) La déstructuration sociale induite par le développement du capitalisme met à l'ordre du jour la question politique de "l'unité intérieure de la nation ».

La bourgeoisie et le prolétariat, les classes montantes appelées à prendre les positions occupées par les Junkers, les propriétaires terriens sont-ils prêts à jouer leur rôle historique, se demande Weber? Sa réponse est négative. Confronté à cette situation historique inédite dans laquelle les principaux acteurs, à ses yeux, n'offrent pas d'alternative politique viable, Weber se tourne vers l'expérience anglaise et française comme source d'inspiration politique. Il retient d'abord de cette expérience deux leçons relatives aux conditions sociales de transformation de ces deux pays en «grandes puissances »: les bourgeoisies anglaise et française auraient su conjuguer leurs intérêts économiques avec leurs ambitions politiques. Elles auraient saisi le lien de dépendance existant entre le développement économique d'une nation et sa position politique comme grande puissance. Ensuite, l'existence «d'une aristocratie ouvrière » dans ces deux pays aurait permis de faire gagner le prolétariat à la cause d'une politique de grande puissance. Weber tire enfin une troisième leçon qui lui permet de définir la tâche incombant aux acteurs intéressés par la transformation politique de l'Allemagne : «l'éducation économique »n'a pas été suffisante pour permettre la prise de conscience des enjeux d'une politique étatique de grande puissance dans ces deux pays. Une éducation 
politique orientée vers la définition des grandes tâches de l'État et la formation politique des citoyens y auraient été nécessaires. Telle serait aussi la voie que les Allemands devraient emprunter (32).

Ainsi, au terme de ses réflexions à partir des enquêtes, Weber relativise fortement la portée politique de l'action des deux associations en la subordonnant au dessein politique qu'il formule, tout en répondant à la fois à la critique du pasteur F. Naumann qui avait jugé que «l'économie politique » comme discipline, décrit plus la réalité qu'elle n'incite à l'action (33).

En 1894, au moment où Weber prononça sa leçon inaugurale à l'université de Fribourg, c'est en termes politiques qu'il percevait encore les clés « d'un développement politique libéral » susceptible d'être emprunté par l'Allemagne.

À notre avis, la découverte des idées de Georg Jellinek sur les conditions culturelles de l'émergence de la démocratie moderne conduiront Weber à repenser cette question. En orientant ses réflexions vers les cultures religieuses, Weber émet une hypothèse relative à l'explication du type de mutation politique vers laquelle il s'est tourné comme champ expérimental : un facteur religieux, le puritanisme en l'occurrence, ne serait-il pas à l'origine de ces transformations? Il s'assigne donc pour tâche de rendre compte de l'existence d'un lien entre puritanisme, démocratie et politique de puissance en cherchant à expliciter les principes d'origine religieuse qui ont permis l'avènement de cet ordre politique. Évidemment, la conséquence de cette réorientation sera la disparition de l'expérience politique française comme référence historique.

\section{Puritanisme, démocratie et politique de puissance : de l'influence de Jellinek}

De nos jours encore, un des nœuds, apparemment gordien, de l'histoire de la réception de la sociologie des religions de Weber réside dans l'appréciation de la portée de l'influence que Jellinek exerça au niveau de l'orientation de cette sociologie. Cependant, de cette appréciation découle l'idée que l'on se fait de la tâche que Weber s'assigna dans cette discipline.

En commençant par l'économie, Weber ne songeait pas reproduire au niveau réflexif des phases du développement historique. Ce point de départ est un effet et une manifestation d'un modèle particulier d'analyse. En ce sens donc, il s'agira pour lui d'effectuer un détour par l'économie pour être à même d'appréhender ces

(32) L'importance du thème de l'éducation politique chez Weber a été soulignée à plusieurs reprises ; cf. Wilhelm Hennis, Wissenschaft vom Menschen, op. cit., pp. 93-113 ; Gerd HufnaGel, Kritik als Beruf. Der Kritische Gehalt im Werk Max Webers, Frankfurt a.M., Propyläen, 1971, pp. 286-291 ; Lawrence A. SCAFF, «Max Weber and Political Education », American Political Science Review, 67, 1973, pp. 128-141.

(33) Friedrich Naumann, Gesammelte Aufsätze, Leipzig, 1894, pp. 42, 48 et 49. 
principes d'origine religieuse qui sont à la base du développement politique auquel il aspire. Pour la démonstration de cette hypothèse, nous reviendrons sur l'importance des idées de G. Jellinek dans le passage de Weber à la sociologie des religions, l'hypothèse centrale et la perspective théorique de Weber dans ce passage, et sur quelques résultats de son investigation.

\subsection{Le passage de Max Weber à la sociologie des religions}

La question du rapport Jellinek/Weber est une question préjudicielle. C'est en raison de la nature de cette question que nous nous sommes autorisé à mentionner le nom de Jellinek dans le titre donné à cet article. Mention dont on aurait pu se passer dans d'autres conditions.

Nous avons déjà eu l'occasion de nous pencher sur cette relation dans un précédent texte. Ce précédent travail permet de tirer deux conclusions, à notre avis, importantes : 1) nonobstant les propres déclarations de Weber sur son bilan scientifique, d'une part, et l'état de la réception de sa sociologie des religions, d'autre part, le rapport puritanisme/démocratie, droits de l'homme, culture politique antiautoritaire a bien occupé une place centrale dans ses recherches ; 2) au sujet de ce rapport, la filiation Jellinek/Weber est manifeste. Toutefois, Weber ne fit pas que réitérer des thèmes ou des thèses énoncés par Jellinek. Il essaya de les traduire dans la perspective théorique de sa sociologie, marquant ainsi ses distances par rapport à la manière jellinekienne de faire l'histoire des idées et des institutions politiques (34). Il faut évidemment se garder de croire que la centralité d'un problème de cet ordre est nécessairement proportionnelle au nombre de pages où il apparaît explicitement. Mais plus encore, alors que dans ce précédent texte, nous croyions à une égale importance des deux axes de recherche (religion/économie) et (religion/ politique), à présent, nous en sommes à démontrer que la signification que Weber a pu accorder au premier est, en fait, dérivée et subordonnée à celle qu'il attribuait au second. Ce faisant, nous avons déplacé l'objet de nos investigations d'un niveau explicite à un niveau qui l'est moins.

Tous ceux qui ont lu Marianne Weber, et qui se sont préoccupés de l'engagement de Weber en sociologie des religions, n'ont pas seulement enregistré l'information selon laquelle Jellinek joua un rôle important dans le développement intellectuel de Weber. Ils ont aussi enregistré le fait que Weber ne répète pas ce dernier dans la mesure où il chercha surtout à explorer un nouveau champ d'investigation. Qu'en est-il de ce champ ? Et de quelle manière s'y est-il pris ? Notre texte n'aurait pas eu de raison d'être si nous n'avions des doutes sur l'apparente évidence des réponses qui ont été apportées à ces questions. Que l'on puisse, ici, très brièvement circonscrire l'enjeu du problème, il n'en demeure pas moins qu'il est décisif.

À défaut d'un texte explicite sur le passage de Weber à la Sociologie des Religions, son lecteur doit se contenter de quelques indications qui ne brillent pas par leur univocité. Une des références majeures qui a servi de source principale à ceux qui se sont préoccupés de cette question est le fragment du discours prononcé par

(34) Cf. Jean Martin OuÉDRAogo, op. cit., pp. 32-48. 
Weber à l'occasion du mariage de la fille de Georg Jellinek et qu'a rapporté Marianne Weber. Entre autres « incitations des plus essentielles » qu'il trouva dans l'œuvre de Jellinek, Max Weber cite « la preuve d'impacts religieux dans la genèse des "Droits de l'Homme" [et] pour la recherche du religieux comme tel dans des domaines où l'on ne la cherche pas de prime abord (35) ». Max Weber fait allusion à l'ouvrage de Jellinek sur la déclaration des droits de l'Homme et du citoyen, écrit en 1895 (36). À partir de cet aveu, les commentateurs en ont conclu que Weber aurait tenté de montrer, dans l'Éthique protestante en particulier, la portée du religieux dans un domaine où on ne l'attend pas du tout, à savoir l'économique. Autrement dit, Weber se serait préoccupé de démontrer dans le domaine économique ce que Jellinek réalisa dans le domaine politique. Il y aurait ainsi un parallélisme entre les travaux des deux auteurs. D'un côté, une réflexion axée sur religion et économie, de l'autre, sur religion et politique (37). La difficulté essentielle qu'introduit cette thèse, c'est qu'elle fait l'impasse sur une donnée incontournable : à savoir que, l'idée d'un lien entre protestantisme et économie, entre « calvinisme », sectes protestantes et « développement du capitalisme », entre « ascèse et vertu bourgeoise » ou enfin celle de l'existence "d'une empreinte ascétique sur le travail professionnel moderne » étaient en fait connues à l'époque de Weber. L'inspiration qu'il eut à la lecture de l'ouvrage de Jellinek ne concerne donc pas ce lieu commun de la recherche historique. Sa préoccupation devrait donc être autre chose qu'un simple retour sur cette question. Comment peut-on néanmoins expliquer que Weber n'ait pas centré d'entrée de jeu son investigation directement sur le rapport entre religion et politique ? qu'il ait procédé d'abord à une incursion dans le domaine économique (38)? Existerait-il un lien quelconque entre les deux orientations de recherche?

Pour comprendre son entreprise, il faut, nous semble-t-il, garder en mémoire les perspectives politiques qu'il défendit antérieurement, notamment dans sa leçon inaugurale. C'est de cette manière que l'on peut saisir l'intérêt que l'ouvrage de Jellinek a pu susciter chez lui. En Angleterre et en France, rappelons-le, Weber voyait à l'œuvre une expérience politique qui offrirait la clé du passage aussi bien d'un État au statut de grande puissance que celui de la bourgeoisie du pouvoir économique au pouvoir politique. Qu'apporte de neuf l'ouvrage de Jellinek ? Quelques idées nouvelles qui marqueront définitivement la propre culture politique de

(35) Marianne Weber, Max Weber. Ein Lebensbild, op. cit., p. 484.

(36) Georg JellineK, Die Erklärung der Menschen und Bürgerrechte. Ein Beitrag zur modernen Verfassungsgeschichte, München - Leipzig, Dunker \& Humblot, 1919, 3 è édition.

(37) Voir le parallèle établi déjà par Paul Honigsheim en 1921 : cf. Paul Honigsheim, « Max Weber als Soziologe. Ein Wort zum Gedächnis », Kölner Zeitschrift für Soziologie und Sozialpsychologie, Sonderheft 7, 1963, p. 83. Reinhard BENDIX, Guenther Roth, eds., Scholarship and Partisanship, Berkeley, University of California Press, 1980, pp. 260-265; Wolfgang Schluchter, Die Entwicklung des okzidentalen Rationalismus, Tübingen, J.C.B. Mohr (Paul Siebeck), 1979, p. 207; idem, Religion und Lebensführung, Vol. 2, op. cit., p. 403, note 52; Hartmut LEHMANN, Max Webers »Protestantische Ethik«, op. cit., p. 15. Hans G. KIPPENBERG, À la découverte de l'histoire des religions, op. cit., pp. 294295.

(38) S.N. Eisenstadt, il y a quelques années, fit la remarque suivante : " The work of Max Weber, although more explicitly focused on the relation between economics and religion, has contained many general and concrete analyses of the interrelations between religion and politics in general and between specific types of religious and political systems in particular »; cf. Shmuel Noah EISENSTADT, Tradition, Change, and Modernity, Londres, John Wiley \& Sons, 1973, p. 169. 
Weber. En effet, elles portent sur l'émergence de la culture et des institutions politiques modernes aux États-Unis. Jellinek impute ce développement aux idées politico-religieuses du puritanisme. La reconnaissance constitutionnelle de la liberté de conscience et d'une manière générale, les Droits de l'Homme, la séparation des Églises de l'État, l'avènement de l'État de Droit, l'égalité des citoyens devant la Loi, etc. sont autant d'acquis imputables au rôle historique des groupes protestants sectaires qui se sont épanouis aux États-Unis. Du point de vue de la protection des Droits de l'individu, les déclarations américaines constituent une grande rupture par rapport aux différents textes juridiques antérieurement promulgués en Angleterre: l'Habeas corpus, la Magna Charta, etc. La déclaration des Droits de l'Homme de la Révolution française a été élaborée sur la base de la réception des différentes déclarations américaines. Les idées politiques qui soustendent cette déclaration ne proviennent ni de la pensée politique de Jean-Jacques Rousseau, ni des théories du droit naturel, ni même de l'Aufklärung, malgré leur importance, mais de celles qu'a impulsées, en dernière analyse, le mouvement protestant sectaire américain. Tel est en résumé, l'apport de Jellinek. Ces idées viennent donc corroborer celles, déjà connues à l'époque, du rôle historique du puritanisme en Occident. Mais à la différence de ces dernières qui mettaient l'accent sur ce rôle dans le domaine économique, celles de Jellinek font découvrir (à Weber aussi) ce rôle dans le domaine politique.

Par rapport à ses vues antérieures, on peut dire que Max Weber découvre l'importance du facteur culturel, politico-religieux en l'occurrence dans les transformations politiques. À partir de cette découverte, il souhaitera élargir le champ de la réflexion de Jellinek dans plusieurs directions. Il projeta d'abord, dès 1904, d'examiner « la situation historique au temps de Cromwell et la doctrine de l'État des Anabaptistes (39) ». En 1904 encore, Jellinek fut invité à (ou se proposa de) parler «des fondements religieux et métaphysiques du Libéralisme » dans le cadre de l'association Eranos (dont Weber était membre). Il n'y a pas de doute que cette intervention de Jellinek a été suivie avec un grand intérêt par Weber (40).

La mise au jour des rapports entre puritanisme et modernité politique par Jellinek a, nous semble-t-il, conduit Weber à reconsidérer ce qu'il entendait auparavant par « expérience » de la bourgeoisie en Angleterre et en France. S'il admet la perspective historique de Jellinek sur la genèse de la modernité politique, il reste cependant encore désarmé par rapport à sa préoccupation principale, à savoir, la question du passage de la bourgeoisie du pouvoir économique au pouvoir politique et les conditions de possibilité d'une politique de grande puissance. Tout au plus sait-il qu'il faudrait dorénavant prendre au sérieux le facteur culturel, le puritanisme en l'occurrence. Il décide donc à son tour de réinvestir le puritanisme. Dans une note de la première édition de l'Éthique protestante, il écrit : « Pour l'histoire de la genèse et de la signification politique de la "liberté de conscience", la "déclaration des Droits de l'Homme" de Jellinek est comme on le sait, fondamentale. Moi aussi, je dois personnellement à cet écrit, l'incitation pour une nouvelle réflexion

(39) Lettre adressée à l'historien Georg von Below le 19. 07. 1904 ; cf. Wilhelm HenNis, Max Weber's Fagestellung, op. cit., p. 205 note 18. Visiblement pour des raisons de compétence, il confia la tâche de cet examen à son ami E. Troeltsch, qui ne nous semble pas avoir répondu à ses attentes.

(40) L’événement est rapporté par Hans G. KippenBerG, op. cit., p. 295. 
sur le puritanisme (41). » On notera ici que Weber parle d'une « réflexion sur le puritanisme ». Il ne dit : ni sur « le puritanisme et le capitalisme » ni sur « le puritanisme et le politique $»$.

Si alors, pour Weber, il s'agit de mettre au jour le lien entre «Puritanisme, démocratie et politique de puissance » comme nous le pensons, pourquoi avoir commencé son étude par protestantisme et économie ? Quel rapport existe-t-il entre une recherche qui porte sur puritanisme et capitalisme et une recherche qui porte sur puritanisme et politique?

\subsection{Hypothèses et perspective théorique}

En abandonnant le champ de l'économie politique comme cadre théorique de réflexion sur ce problème, Weber maintient, certes, la préoccupation qui l'avait conduit à se tourner vers l'expérience historique de la France et de l'Angleterre ; mais, à la différence de ses textes antérieurs, la référence à la France disparaît. Audelà de l'auto-justification de Weber, pourquoi l'Angleterre, lui a-t-elle servi de référence politique, peut-on enfin se demander ? Deux raisons ont été avancées que l'on pourrait en effet retenir : son anglophilie avait d'abord un fondement familial. À Manchester et à Londres vécurent et prospérèrent comme entrepreneurs une partie de ses ancêtres. À cela s'ajouta l'importance qu'acquit l'Angleterre comme modèle, dans l'imaginaire politique des libéraux allemands : "Depuis la période napoléonienne, rappelle G. Roth, l'Angleterre avait été, pour beaucoup de libéraux allemands, le "grand frère" qui pouvait montrer la voie hors des confusions de l'histoire française et des frustrations de l'histoire allemande. Après l'unification allemande de 1871, le nombre des admirateurs libéraux déclina progressivement jusqu'au moment où vint le défi fatal de l'Allemagne à l'Empire britannique. Max Weber était l'un des derniers à considérer l'Angleterre comme un modèle [en matière] de réforme constitutionnelle et de Weltpolitik [politique de grande puissance] (42). » À propos de la France, il existe une raison d'ordre strictement intellectuel à ne pas sous-estimer: Weber s'est implicitement réapproprié la thèse de Jellinek sur la place de ce pays (et des «pays latins » d'une manière générale), par rapport à l'Amérique, dans l'avènement des libertés modernes (43).

(41) Max Weber, "Die protestantische Ethik und der "Geist" des Kapitalismus », Archiv für Sozialwissenschaft und Soziapolitik, 20, 1904, p. 43, note 38.

(42) Guenther Rотн, «Weber: the would be Englishman, op. cit., p. 83. Idem., «Zur Entstehung und Wirkungsgeschichte von Webers "Protestantische Ethik" ", op. cit. Voir également Gustav SCHMIDT, Deutscher Historismus und der Übergang zur parlamentarischen Demokratie, Lübeck, Matthiesen, 1964, pp. 47 sq, $226 s q$. Comme l'a remarqué à juste titre Wolfgang J. Mommsen, la signification du modèle anglais dans la pensée politique de Max Weber ne doit pas être surestimée. Cf. Wolfgang J. Mommsen, Max Weber und die deutsche Politik, op. cit., p. 446.

(43) Quant à l'invocation de l'importance des «principes de 1789 » dans sa lettre au comte Keyserling du 26 juin 1911, Weber reprend simplement le point de vue de Jellinek sur le rôle de la Révolution française dans la diffusion, en Europe, des idées relatives aux droits de l'Homme, initialement mises en œuvre sur le plan constitutionnel en Amérique. Cf. Eduard BAUMGARTEN, Max Weber, Werk und Person, Tübingen, J.C.B. Mohr (Paul Siebeck), 1964, p. 429. Voir aussi ÉP, pp. 81 et 83. Sur le point de vue de Jellinek, voir Georg JeLlineK, Die Erklärung, op. cit., pp. 71-72. 
Il faut rappeler que l'Éthique Protestante commence par une mise au point fondée sur des données statistiques concernant le lien, en Allemagne, entre la stratification sociale, les confessions religieuses, le niveau de formation et les activités professionnelles. Se référant à des études existantes, Max Weber fait apparaître que les possesseurs de capitaux, d'entreprises, la couche supérieure des travailleurs qualifiés, le personnel technique et commercial «sont en grande majorité protestants " c'est-à-dire luthériens dans ce pays (44). Il réitère ainsi un des points essentiels de son analyse antérieure sur le développement du capitalisme en Allemagne. En mettant l'accent à présent sur la dimension culturelle qui lui pose problème, il précise par la même occasion l'objet de son interrogation antérieure : la question de la transformation politique de l'Allemagne post-bismarckienne et la nécessité d'une assimilation de l'expérience anglaise en matière de culture politicoreligieuse. C'est ainsi qu'il aborde cette expérience avec une hypothèse qu'il formule et un modèle d'analyse qu'il met en œuvre.

La leçon politique qu'il avait cru tirer de l'expérience anglaise ne lui apparaît plus évidente. La question de la particularité du développement politique de ce pays mérite désormais, à ses yeux, un examen plus minutieux. Pour poser le problème dans toute sa rigueur, Weber prend appui sur Montesquieu : «Dans l'esprit des Lois (XX, chap. 7), Montesquieu dit des Anglais : "de tous les peuples du monde, c'est celui qui aurait apporté le plus de ces trois grandes choses: la piété, le commerce et la liberté". Leur supériorité dans le domaine du commerce et - ce qui relève d'un autre contexte - leur appropriation d'institutions politiques libres, ne dépendraient-elles pas, peut-être, de ce record de piété que Montesquieu leur reconnaît (45) ? » Weber y revient plus tard en précisant du même coup la particularité du champ de l'expérience historique qui lui importe. En effet, à la différence des pays latins, note-t-il, en Angleterre, «1) le puritanisme a permis à ses adeptes de créer des institutions libres et [à ce pays] de devenir une grande puissance (Weltmacht), et 2) il a transformé cette "calculabilité" [...] qui est en fait constitutive du capitalisme, de moyen économique en principe de conduite de vie global » (46). Ce développement n'est pas à imputer au "caractère national » des anglais, mais à ce facteur culturel qu'est le puritanisme (47). Et c'est même plutôt ce facteur, en particulier, qui permet de rendre compte du caractère national des peuples qu'il a marqué de son sceau (48).

Le retour à l'expérience anglaise a donc pour terme la formulation d'un problème historique qu'il se propose d'investir : l'origine culturelle de la transformation des institutions de ce pays dans un sens libéral et de sa politique étatique en politique de puissance. À ses yeux, ce développement serait imputable à la culture religieuse puritaine. La validité de cette hypothèse historique n'est pas réductible à

(44) ÉP, p. 31.

(45) Ibid, pp. 43-44; (traduction modifiée).

(46) Ibid, p. 207, note 10. [...] de nous ; (traduction modifiée).

(47) Ibid., p. 104 : «D'abord, le pouvoir des mouvements religieux, écrit-il, - non pas uniquement, mais en premier lieu - qui a créé ici ces différences que nous ressentons encore aujourd'hui »; (traduction modifiée) ; cf. également ibid., p. 252.

(48) Ibid., p. 123. Voir également ibid., pp. $203 ; 237$ note $83 ; 239$. Rétrospectivement, en 1913 , Weber considéra sa leçon inaugurale « immature » à beaucoup d'égards. À notre avis, il ne percevait pas encore ce facteur religieux à l'époque, au moment où il invoquait l'expérience de l'Angleterre. Cf. Marianne Weber, Max Weber, op. cit., p. 416. 
ce domaine. Elle permettrait également de rendre compte du développement économique de ce pays. En ce sens donc, il ne s'agissait pas pour lui de revenir simplement sur les travaux relatifs au rapport entre protestantisme et capitalisme. Il formule également d'autres hypothèses.

C'est de manière anecdotique et ramassée que Weber propose, en fait, une seconde hypothèse concernant le rapport du calvinisme à l'économie (profession) et au politique, au moment où il entend préciser l'idéaltype de "l'esprit du capitalisme". Benjamin Franklin rapporta dans son autobiographie une citation de la Bible que son père, " un strict calviniste », n'aurait eu de cesse de lui répéter dans sa jeune enfance : "Vois-tu un homme actif dans sa vocation, il fera face à des rois (49). " Max Weber relate cette anecdote à propos d'une des implications de la morale de Franklin. Afin de préciser cet idéaltype, il se servit d'un document émanant de ce dernier. À l'aide de ce document, il suggère que " l'acquisition de l'argent est - dans la mesure où elle se fait de manière licite - au sein de l'ordre économique moderne, le résultat et l'expression de l'application dans la profession; et cette application est, on s'en rend compte sans difficulté, l'alpha et l’oméga véritables de la morale de Franklin [...] (50) ». Selon cette morale, l'acquisition est devenue le but de la vie de l'homme et non plus un moyen pour satisfaire ses besoins matériels. Dans cet esprit, " on doit (ou voit? vérifier) "des hommes faire de l'argent" (51). " Cependant, note Weber, cette morale, bien qu'elle paraisse absurde, contient des sentiments liés « intimement à certaines représentations religieuses ». En effet, lorsque Franklin était confronté au soupçon d'absurdité de cette morale, il répondait en donnant la citation biblique apprise de son père.

(49) ÉP, p. 52. (traduction modifiée). La citation de Weber est incomplète et partiellement déformée: incomplète au regard de la référence biblique originaire donnée par B. Franklin dans ses mémoires (à laquelle Weber renvoie en note) et déformée au regard de la traduction allemande des mémoires en question dont il s'est apparemment servi. À la suite d'une découverte récente, nous savons que Weber était en possession de la traduction allemande des mémoires de Franklin. Cf. Jean Martin OuÉDraogo, op. cit., p. 33, note 33. Le passage traduit par B. Auerbach est le suivant : « siehest Du einen Mann redlich ("rüstig" chez Weber) in seinem Beruf, so soll er vor Königen stehen ; er soll nicht vor gemeinen Leuten stehen »; cf. Benjamin FrankLin, Sein Leben, vom Ihm selbst geschrieben, (mit einem Vorwort von B. Auerbach und einer historish-politischen Einleitung von F. Kapp), Stuttgart, Auerbach, 1876, p. 278. Voir Max WeBer, Die Protestantische Ethik und der " Geist des Kapitalismus ", Weinheim, Beltz Atenäum, 1996, pp. 15-16, note 25. La citation originale est la suivante : « Seest thou a Man diligent in his calling, he shall stand before Kings, he shall not stand before mean Men »; cf. Benjamin Franklin, Autobiography and Other Writings, Oxford, Oxford University Press, 1993, p. 81. Une traduction française de la plume d'Édouard Laboulaye de cette autobiograhie, parue une décennie plus tôt, restituait en ces termes le passage biblique en question : « avez-vous vu un homme ardent à l'ouvrage ? C'est auprès des rois qu'il se tiendra, et non parmi la foule »; cf. idem, Mémoires, écrits par lui-même, traduits de l'anglais et annotés par Édouard Laboulaye, Paris, Librairie de L. Hachette et $\mathrm{C}^{\mathrm{ie}}$, 1866, p. 155. Dans le texte de Weber, nous trouvons donc une référence exacte (Prov. XXII, 29) mais qui renvoie à un passage tronqué et partiellement déformé ! Cette intervention de Weber induit, nous a-t-il semblé, un sens politique plus prononcé du proverbe. À noter toutefois que B. Franklin avait pris soin de mettre en garde son lecteur contre toute interprétation littérale du proverbe en question. Cf. Ibid. J. Chavy, le traducteur du texte de Weber en français, a cru devoir restituer le proverbe en question, en le citant tel qu'il est donné plutôt dans la bible de Luther, cf. ÉP, p. 52 note 9. Alors que Weber cherchait à montrer que le remplacement du mot «besogne » (« Geschäft») (apparaissant encore chez Luther) par celui de «vocation» («Beruf») («calling») (chez les puritains) est justement symptomatique de la nouvelle culture religieuse qu'ils impulsèrent.

(50) Ibid., p. 52 ; (traduction modifiée).

(51) Ibid. 
L'intérêt de cette anecdote pour Weber réside, nous semble-t-il, dans le fait qu'elle signale l'existence d'un lien implicite entre calvinisme (la culture religieuse du père de Franklin), la vocation dans le monde économique et une certaine attitude politique. Car, pour cette culture, celui qui se montre appliqué dans sa profession, désormais conçue comme une vocation, est, pour ainsi dire destiné à pouvoir relever la tête devant des rois. Rendre ce lien explicite du point de vue sociologique, tel est donc le programme que se fixe Weber. À partir de cette indication sur la culture politico-religieuse d'un émigré anglais de la Nouvelle Angleterre, Weber pensera détenir le fil rouge qui le conduirait non seulement au pays d'origine de cet émigré, à savoir l'Angleterre, mais dans le même mouvement, à comprendre le rapport de cette culture religieuse au politique, à partir de celui qui le noue à la profession, c'est-à-dire aussi à l'économie (52).

Ces hypothèses ne sont toutefois pas les seules qui commandent l'ensemble de ses études sur les religions amorcées avec l'Éthique protestante. Pour centrales qu'elles soient, elles ne permettent pas à elles seules de comprendre ce travail de " réinterprétation de la signification historique du puritanisme » (G. Roth) (53).

Une troisième hypothèse, dérivant de la seconde, mais différente d'elle parce que de portée plus générale, est celle selon laquelle, le rapport du puritanisme (et d'une manière générale de toute religion) au monde est le résultat d'une position qui est fondamentalement unitaire. Qu'est-ce que le monde dans cette optique ? Le monde (Welt) chez Weber, c'est d'abord et initialement la triade : profession - État - famille. Dans les Considérations intermédiaires notamment, il parlera des ordres (Ordnungen) et valeurs (Werte) du monde ou encore d'ordres de vie (Lebensordnungen), élargissant ainsi son champ, en subsumant sous cette notion de monde, la science, l'art, et l'intellectualisme (54). Par ailleurs, la triade primaire constitutive du monde ne représente pas nécessairement des réalités univoques. En effet, la profession renvoie aussi à l'économie, l'État au politique, tandis que la famille renvoie aussi à la sexualité. En vertu de cette troisième hypothèse, le rapport d'une religion à la profession est aussi celle qu'elle entretient par exemple avec la famille et avec l'État. Par conséquent, lorsque l'on a une connaissance de la position d'une religion par rapport à l'un des éléments de la triade, l'on détient ipso facto des indications sur sa position vis-à-vis des autres éléments (55). De ce point de vue, les rapports de tension avec le monde, de rejet du monde, d'indifférence au monde, d'adaptation au monde, etc. se tissent conformément à une position unitaire. De même, la possibilité d'une transformation d'origine religieuse du monde dérive d'une même position. Dans une perspective comparative, le rapport au monde du puritanisme possède, selon Weber, un caractère qui lui est spécifique. En effet, à la différence des autres cultures religieuses, le rapport au monde du puritanisme est une conséquence de celui qui le noue à la profession et donc à l'économique. Si bien que l'on pourrait dire - en utilisant ici un langage marxiste - que ce rapport au monde est en dernière analyse déterminé par un rapport initial à l'économique. Ainsi, l'attitude que le puritanisme (calvinisme) imprègne au domaine professionnel

(52) Sur l'origine de la famille Franklin, voir Benjamin Franklin, Mémoires, op. cit., pp. 16 et sq.

(53) Guenther Rотн, "Weber the Would-Be Englishman: Anglophilia and Family History », op. cit.

(54) $S R$, pp. 410-460.

(55) ÉS, pp. 621, 543-544 et 585-632. 
aurait une incidence sur l'attitude politique et sur la conduite dans le monde et ses ordres en général.

Une quatrième hypothèse historique, de portée générale, corollaire de la première concernant le rôle du puritanisme en Angleterre, intervient également. Cette dernière est relative au poids historique du facteur religieux dans les sociétés prémodernes ou non modernes. "La signification pratique de la culture [religieuse] était plus importante dans le passé qu'elle ne l'est tout au moins de nos jours, écritil (56) ». Les contenus de la conscience religieuse concernaient aussi bien «la conduite de vie », « la culture » que « le caractère national ». Dans le contexte du monde moderne, au regard de la place qu'y occupent les croyances religieuses, un tel fait sociologique de grande portée est susceptible (à l'époque où vivait encore Weber) d'être sous-estimé (57).

Au total, nous avons donc deux hypothèses générales concernant les cultures religieuses et deux hypothèses corrélatives, spécifiques au puritanisme. La perspective théorique à partir de laquelle Weber a envisagé le puritanisme et d'une manière générale les religions, a gagné véritablement en clarté seulement tardivement. En effet, chemin faisant, des précisions d'ordre méthodologique et épistémologique émaillaient sans cesse sa réflexion. Il faut attendre 1913 avec l'article publié dans Logos sur "quelques catégories de la sociologie compréhensive » pour lire une mise au point importante de cette perspective. Il reprend ce texte et le publie comme chapitre premier de son ouvrage Économie et Société (58). Du début à la fin, sa perspective est demeurée invariable sur la manière dont les idées religieuses deviennent "efficaces dans l'histoire». Des nombreuses formulations qu'il en donne, celle que nous pouvons lire dans l'Introduction à l'Éthique économique des religions mondiales (1915) est assez limpide : "Ce sont les intérêts (matériels et idéels) et non les idées qui gouvernent l'action des hommes. Toutefois, "les images du monde", qui ont été créées par le moyen "d'idées", ont très souvent joué le rôle d'aiguilleurs, en déterminant les voies à l'intérieur desquelles la dynamique des intérêts a été le moteur de l'action. C'est en fonction de l'image du monde, en effet, qu'était déterminé « de quoi » et en vue de quoi l'on voulait et - ne l'oublions pas - l'on pouvait être "délivré” (59). " Sa position par rapport à Marx dérive de cette perspective.

Weber a réinterprété le savoir disponible de son temps en fonction de ses préoccupations du moment et à partir de cette perspective théorique (60). Ceci est aussi valable à propos de la manière dont il tenta d'intégrer l'apport de Jellinek. Sur ce point, il chercha à traduire la perspective historique de Jellinek sur l'avènement de la modernité politique en perspective sociologique. Cette traduction concerne aussi bien l'appareil conceptuel (secte et Église par exemple), la démarche du point de vue théorique que l'agenda scientifique de sa sociologie. Il ne cessa d'insister sur le changement de point de vue entre l'approche strictement historique et l'approche sociologique. L'opposition récurrente entre « historique »

(56) Ibid., p. 533 ; (traduction modifiée). voir également ibid. p. 315.

(57) ÉP, p. 252.

(58) Max Weber, Essais sur la théorie de la science, Paris, Plon, 1965, pp. 325-398. ÉS, pp. 3-59.

(59) $S R$, pp. 349-350 ; (traduction modifiée).

(60) ÉP, p. 25. 
(historisch) et «par principe» (prinzipiell) dans l'analyse du rôle historique des sectes et autres associations recouvre en fait ce changement de perspective (61). La recherche sociologique doit se tourner vers l'exploration de l'impact des associations (au sens sociologique) en général sur les conceptions du monde (Weltanschauungen), les domaines politiques, l'opinion publique, les valeurs culturelles, etc., c'est-à-dire sur des phénomènes sociaux que l'on ne soupçonne pas avoir été marqués par ces associations (62).

On comprend donc pourquoi Weber a envisagé un retour au puritanisme. Mais, il n'abordera pas de manière frontale le lien entre puritanisme et politique. Conformément à ses hypothèses, ses recherches sur religion et profession (économie) d'une manière générale ne pouvaient donc constituer qu'un détour. Mais cet itinéraire, espérait-il, devait le mener à comprendre le rapport entre puritanisme et politique, c'est-à-dire, en particulier, son rapport à l'avènement des institutions politiques démocratiques, et de la politique de grande puissance en Angleterre, qui, dans son esprit, portent la marque politique de la bourgeoisie. Parvient-il effectivement à mettre au jour cette relation dans ses travaux ?

\subsection{La double stratégie de recherche de Max Weber}

Commençons ici par faire une série de remarques :

1) De l'Éthique protestante à la rédaction de l'avant-propos qui clôt les trois volumes sur l'Éthique économique des religions mondiales, nous ne trouvons pas trace d'un texte dont le titre serait «Puritanisme, économie, institutions politiques et politique de grande puissance en Angleterre ", projet annoncé au début de son étude à l'aide d'une référence à Montesquieu, quoique, à quelques reprises, des notes rappellent bien la permanence de sa préoccupation (63). En fait, Weber tentera de traiter ce thème de manière particulière.

2) En 1905 encore, à la fin de l'Éthique protestante, Weber songea prolonger son étude par une recherche sur la signification du rationalisme ascétique, c'est-à-dire du puritanisme, par rapport à l'éthique politico-sociale des communautés sociales (des conventicules à l'Etat) aussi bien au regard de leur genre d'organisation que de leur fonction (64). Dans la deuxième version du texte, il signale en note avoir renoncé à poursuivre ce programme de recherche d'une part, en raison de la parution du livre de Troeltsch sur les Soziallehren, qui le dispensa de cette tâche et d'autre part, parce qu'il décida entre-temps de prolonger ses recherches antérieures par des travaux comparatifs sur les cultures religieuses mondiales (65).

(61) Voir Max Weber, Gesammelte Aufsätze zur Soziologie und Sozialpolitik, Tübingen, J.C.B. Mohr (Paul Siebeck), 1924, pp. 442 sq.; SR., p. 327 ; également, Jean Martin OuÉDrAOGO, op. cit., pp. 35 et $s q$.

(62) Max Weber, Gesammelte Aufsätze zur Soziologie und Sozialpolitik, op. cit., pp. 444 et 445. Voir aussi idem., Die protestantische Ethik II. Kritiken und Antikritiken, Gütersloh, GTB Siebenstern, 1982, pp. 173-174; en abrégé, $P E$.

(63) ÉP , pp. 192, note $179 ; 207$, note $10 ; 232$, note 73 . La lettre adressée au comte Keyserling du 21 juin 1911 s'inscrit aussi dans l'esprit de ces notes; cf. Eduard BAuMgarten, Max Weber. Werk und Person, op. cit.

(64) $P E$, p. 251.

(65) $P E$, p. 253 , note 115. 
3) En 1919, dans le bulletin d'information de la maison d'édition Mohr, Max Weber eut l'occasion de donner un aperçu général de ce qu'il réalisa avec les trois volumes portant le titre L'Éthique économique des religions mondiales. Il explique y avoir cherché à rendre compte de la singularité économique et sociale de l'Occident, de sa genèse et de son rapport à l'éthique religieuse (66). Par ailleurs, dans la première note introductive à la seconde version de l'Éthique protestante qui représente le premier des trois volumes, il déclare n'avoir rien modifié d'essentiel dans son texte (67). Au sujet du rapport religion/politique, une comparaison des deux versions laisse cependant apparaître des modifications importantes dans la seconde version de son texte. Plusieurs ajouts ou suppressions de phrases ont été faites dans la deuxième mouture, indiquant dans l'ensemble, soit que la discussion détaillée du rapport religion et politique ne pût trouver de place dans ce texte, soit que l'objet du texte concernât essentiellement le lien entre le développement du rationalisme économique et l'éthique puritaine (68). Ce jugement rétrospectif de 1919, par sa modestie, manifeste, à notre avis, les difficultés que Weber rencontra dans la réalisation de son projet. Car, il semble avoir revu sa signification à la baisse, non seulement par rapport à ce qu'il annonça au début de son étude, mais aussi de ce qu'il prétendit, en pleine controverse, avoir mis en lumière : la portée de l'idée de la vocation dans la profession au-delà de la sphère économique.

Ce faisant, Max Weber contribuera personnellement à orienter la réception de son texte de façon déterminante dans le sillage des discussions sur le rapport entre religion et économie. Pourtant ces modifications n'ont pas éclipsé ce que nous considérons comme étant sa préoccupation centrale dans ces textes. En 1910, en effet, en pleine controverse sur la validité de l'Éthique protestante, Weber laisse entrevoir encore la portée de l'idée de la vocation comme devoir au-delà de la sphère de l'économique. "Enfin, écrit-il, cette composante de "l'esprit" du capitalisme des Temps modernes que j'ai spécialement analysé - l'idée du "devoir professionnel" avec tout ce qui lui est associé - se trouve en revanche au sein de l'économie portée par "l'esprit" du capitalisme (au sens général de l'expression) seulement dans une période historique déterminée ; par ailleurs, cette composante dépasse le domaine de l'économie pour atteindre des sphères tout à fait hétérogènes de l'action humaine. (69) » Autrement dit, l'idée du devoir professionnel, audelà de sa portée économique concerne aussi bien l'action politique, l'action dans la famille, ou encore l'attitude face à la sexualité, etc. La signification du développement de l'homme professionnel, insiste-t-il auprès de ses contradicteurs - qui

(66) $M W G, \mathrm{I} / 19$, p. 28

(67) ÉP, p. 30, note 17 ; voir aussi ibid., p. 253, note 115. Cf. l'édition de la première version réalisée grâce aux soins de Klaus Lichtblau et de Johannes Weiß; idem, Die Protestantische Ethik und der "Geist des Kapitalismus », op. cit. D’une manière générale, les modifications intervenues dans la deuxième version ont été signalées par les deux éditeurs à l'aide de crochets [ ] numérotés. Pour la comparaison des deux versions à propos du problème qui nous intéresse ici, nous nous reporterons à ces indications. La référence de cette première version sera abrégée par la suite par PEI.

(68) Déjà, à propos du traitement de la question de «l'appropriation des institutions politiques libres » chez les Anglais en référence à Montesquieu : il existe bien une différence entre « ce qui relève d'un autre contexte » (seconde version) et « ce que nous aborderons bien encore dans un autre contexte » (première version). Cf. $E P$, p. 43 et $P E I$, p. 10 (cette modification n'a pas été signalée). Ensuite, $P E$, p. $198 ; P E I,[341]$, p. $118 ; E ́$, p. 205 , note $4 ; P E I,[353]$, p. $193 ; E ́ P$, p. 215, note $33 ; P E I$, [368], p. 131.

(69) PE II, p. 173. (souligné par nous). 
réduisaient sa recherche uniquement à cet aspect -, ne devrait pas être limitée à celle qu'il possède en tant que composante de l'esprit du capitalisme (70).

Quel que soit le propre jugement de Weber sur les résultats de ses investigations, ces résultats méritent d'être revisités, ne serait-ce que pour leur valeur strictement historique. Ses réflexions sur le rapport entre puritanisme et politique en particulier relèvent de son œuvre au même titre que n'importe quel texte issu de sa plume.

Que peut-on retenir de ces investigations qui l'ont mobilisé, au plus haut point, nous semble t-il ? Pour investir le puritanisme, Weber adoptera une double stratégie de recherche, inspirée par les hypothèses mentionnées ci-dessus, qu'il poursuivra tout au long de ses travaux sociologiques sur les cultures religieuses.

\subsubsection{De la doctrine de la grâce}

Du point de vue théorique, en ce qui concerne en particulier l'importance qu'il accordera à la doctrine de la grâce dans le puritanisme et, de façon générale, dans ses recherches sur les cultures religieuses, Weber ne s'explique véritablement que plus tard; c'est-à-dire, au moment où il songe à une analyse comparative des cultures religieuses et à la systématisation de ses recherches (71). Nous découvrons alors en effet, une thèse importante, explicitement formulée, mais qui se trouvait déjà en filigrane dans ses réflexions sur l'apport de Luther, au moment où il commence l'Éthique protestante. Cette thèse précise la manière dont il perçoit l'efficacité sociale des idées religieuses. L'influence d'une religion sur la conduite de vie de ses adeptes, nous apprend-il, est fonction des conditions d'une nouvelle naissance (Wiedergeburt) qu'elle dicte à ces derniers. Car, il n'y a de salut que pour celui qui accepte de renaître conformément à ces exigences religieuses. Cette nouvelle naissance dépend aussi bien de la méthode de rédemption (Erlösungsweg) à suivre par l'adepte, que de la qualité psychique de la possession de salut (Heilsbesitz) recherché (c'est-à-dire des signes d'espérance) (72).

La voie de rédemption (ou de salut), dont il est question dans le puritanisme, fait appel à la théorie de la prédestination. Celle-ci exclut les voies qui font appel à l'œuvre de l'individu : que celle-ci soit personnelle (bouddhisme primitif), ou se présente sous la forme de cultes ou de rites (hindouisme), ou de prestations sociales (hindouisme, catholicisme), ou les voies qui recourent à la contemplation mystique ; ou encore celles qui se conçoivent comme résultats, soit de l'attribution d'un excédent de grâce que possèderaient certains individus (sauveurs), ou qui émanerait d'une institution (catholicisme) ; ou, enfin, celles qui font appel à la foi (christianisme primitif, luthéranisme). La dévaluation radicale de toute forme de

(70) Ibid.

(71) C'est-à-dire, dans l'Éthique économique des religions mondiales et dans le texte posthume sur les types de communalisation religieuse (chap.V) d'Économie et Société. Voir note 3.

(72) ÉS, pp. 545 ; 554. Nous avons opté de traduire "Wiedergeburt " par «nouvelle naissance " plutôt que par renaissance. Nous remercions Jean Séguy d'avoir attirer notre attention sur l'ambiguïté de l'ancienne traduction. Sur l'application du modèle d'analyse à la sociologie de la Bible, voir Jean Martin OuÉdraogo, «Esquisse d'une sociologie de la Bible chez Max Weber », Social Compass, 46, (4), 1999, pp. 409-439. 
distribution de la grâce, quelle soit magique, sacramentelle, institutionnelle a été la conséquence de cette doctrine (73).

La rédemption du puritanisme fait appel à un Dieu transcendant, omniscient et omnipotent. Ce Dieu distribue la grâce gratuitement à quelques élus seulement. L'humanité se trouve ainsi composée d'élus et de damnés de toute éternité. Ces décrets sont tout autant insondables qu'immuables. De toutes les voies de salut, la doctrine de la prédestination est, selon Weber, celle qui garantit le plus l'assurance du salut, dès lors que l'individu est persuadé d'être en sa possession (74). Car, plutôt que de conduire ses adeptes vers le fatalisme, cette doctrine, en devenant une religiosité de masse, connaîtra une inflexion qui retentira dans le rapport de ses adeptes à l'action. En effet, dans sa pureté, cette doctrine est intenable du point de vue psychologique. «L'incertitude absolue (face à l'élection) est insupportable à la longue. (75) » L'inflexion se traduira dans la possibilité offerte à l'adepte de lire dans sa vie quotidienne des signes d'espérances, "des symptômes" de son élection. «Or, étant donné que Dieu a, en quelque sorte, condescendu à révéler quelques commandements positifs concernant la façon de vivre qui lui est agréable, ces symptômes ne peuvent résider - comme pour tout charisme religieux actif - que dans la confirmation décisive que l'individu est capable de participer à leur accomplissement en tant qu'instrument de Dieu, et cela continûment et méthodiquement, car on possède la grâce ou toujours, ou jamais. (76) »

Toute religion entretient un rapport unitaire au monde. Celui qui caractérise le puritanisme (ou l'ascèse intramondaine) tire son origine de la "certitudo salutis” (77). L'auto-perception des virtuoses religieux (comme Weber les appelle) comme instruments de Dieu les conduit à soumettre leurs pulsions naturelles à une conduite de vie systématique, et leur rapport à la vie sociale à une critique éthicoreligieuse radicale. L'accomplissement de sa qualité en tant qu'instrument élu de Dieu se fera à l'intérieur et en opposition au monde. Le virtuose religieux devient ainsi un réformateur ou un révolutionnaire comme l'ont illustré le parlement des saints sous Cromwell, l'État quaker ou encore les conventicules piétistes radicaux (78). Plutôt qu'une attitude fataliste résultant de la doctrine de la prédestination, on constate un comportement orienté vers l'action. Deux idées vont contribuer à cette orientation : celle selon laquelle le chrétien ne peut avoir la confirmation de son élection qu'en agissant pour la gloire de Dieu ; et celle selon laquelle toute idolâtrie de la créature et tout attachement à des relations personnelles détournent de Dieu. En créant le monde et l'ordre social, Dieu y aurait voulu comme moyen de célébration de sa gloire ce qui est « objectivement adéquat». Le chrétien qui se préoccupe donc d'avoir les preuves de son élection agit conformément aux desseins de Dieu qui ne peuvent être qu'impersonnels. Le puritanisme se dotera ainsi d'une force psychologique qui lui confèrera une capacité d'initiative en matière organisationnelle dont la portée se situe au-delà de l'organisation de la communauté ecclésiale. Le monde dans son ensemble est concerné. « Le [monde]

(73) $E S$, p. 582.

(74) ÉS, p. 581. Voir aussi ÉP, pp. $118 s q$.

(75) Ibid.

(76) Ibid. Voir aussi pp. $130 \mathrm{sq}$.

(77) ÉS, p. 621.

(78) ÉS, p. 555. 
demeure comme créature, dévaluée : un abandon visant la jouissance de ses biens met en péril la concentration sur le bien de salut et sa possession, et serait symptomatique d'une conviction non sainte et de l'absence d'une nouvelle naissance. Mais le monde est, en tant que création de Dieu - dont le pouvoir s'y manifeste en dépit de son état de chose créée - l'unique matériau sur lequel le charisme religieux personnel doit se confirmer par un agir éthique rationnel, afin d'être certain de son état de grâce et de le demeurer. Comme objet de cette confirmation active, les ordres du monde dans lesquels l'ascète est placé vont devenir une "vocation" à accomplir rationnellement. La jouissance de la richesse est alors prohibée ; - "la vocation" cependant, c'est l'économie conduite de manière rationnelle, éthiquement ordonnée et dans la stricte légalité; le succès [qui en résulte], donc l'acquisition manifeste la bénédiction divine du travail du pieux et par conséquent, le caractère agréable à Dieu de sa conduite de vie économique est [ainsi] rendu visible. Prohibée est toute exubérance de sentiment envers les êtres humains en tant qu'idolâtrie de la créature niant la valeur exclusive des dons divins de salut; "la vocation" cependant, c'est la sobre participation aux finalités objectives et légales, conçues par Dieu, des groupements à but déterminé [au sein] du monde. Prohibé est l'érotisme divinisant la créature; la vocation voulue par Dieu, c'est une "sobre procréation d'enfants" dans le mariage (comme l'expriment les puritains). Prohibée est la violence que l'individu exerce sur ses semblables, que ce soit par passion, par soif de vengeance ou pour des motifs personnels; est voulue par Dieu en revanche la répression rationnelle et le châtiment des péchés et de la désobéissance dans un État organisé à cette fin. Prohibée est l'idolâtrie de la créature qu'est la jouissance personnelle et mondaine du pouvoir ; voulue par Dieu est la domination de l'ordre rationnel de la Loi. "L'ascète intramondain" est un rationaliste aussi bien dans le sens de la systématisation rationnelle de sa propre conduite de vie personnelle que dans le sens du rejet de tout ce qui est irrationnel du point de vue éthique, que ce soit ce qui est artistique, ou que ce soit les sentiments personnels au sein du monde et de ses ordres (79). " Le besoin d'action (Tatendrang) que libèrera cette doctrine de la grâce orientera de cette manière cette culture religieuse vers une rationalisation du monde historique. Le règne de Dieu ne se limite pas à la sphère de l'organisation religieuse, il doit s'étendre à la société dans sa globalité. L'immunité contre le césarisme qui caractérise les peuples au passé puritain, le scepticisme des puritains à l'égard de tout pouvoir séculier et de toute légitimité (d'où l'image qu'ils donnèrent partout d'être dangereux pour l'État et pour toute légitimité), leur hostilité au style de vie et au mode de reproduction sociale de type féodal (à l'opposé du "self made man" qu'ils valorisent) sont autant de manifestations de cette culture politique anti-autoritaire que Max Weber identifie au puritanisme (80).

Luther avait introduit en son temps la notion de vocation ("Beruf"), attribuant ainsi une valeur positive au travail séculier, qui devenait l'expression de "l'amour du prochain". Ceci constituait une nouveauté dans l'histoire des religions et pas seulement par rapport à l'histoire du christianisme. Mais, dans la mesure où, les « œuvres » ne devinrent jamais le lieu de reconnaissance de la nouvelle naissance

(79) ÉS, pp. 556-557. (Trad. modifiée). Voir aussi plus loin ibid. pp. 557-558; 567-568; et ÉP, p. 126 et notes 32 et 33 p. 128.

(80) ÉS, pp. $568 ; 582 ; E ́ P$, p. 220. Voir Jean Martin OuÉdraogo, « Sociologie religieuse et modernité politique chez Max Weber », op. cit., pp. $36 \mathrm{sq}$. 
et surtout, dans la mesure où, "l'habitus émotionnel de se savoir à l'abri sous la bonté et la grâce de Dieu demeura la forme prédominante de la certitude de salut, l'attitude envers le monde demeura aussi une "résignation" patiente dans les ordres de celui-ci [...] Il manque au luthéranisme toute impulsion dans le sens d'une attitude révolutionnaire sociale ou politique ou même simplement réformiste rationnelle. Il s'agit [pour lui] de préserver les biens de salut de la foi dans le monde et contre lui et non de le transformer de manière éthique et rationnelle (81). » Le rapport logique et psychologique entre les idées de Dieu, du monde et la position vis-à-vis de ce monde, lui sert en même temps de fondement pour comprendre le rapport du puritanisme (contrasté à dessein avec le luthéranisme) au monde historique (82).

\subsubsection{De la conception de l'organisation ecclésiale}

Parallèlement à cette première stratégie de recherche, Weber en poursuivra une seconde qui le conduira à découvrir d'autres connexions entre puritanisme et politique. À cette occasion cependant, le puritanisme apparaîtra plus différencié. Contrairement au calvinisme qu'il avait privilégié au départ, l'univers du protestantisme sectaire (baptiste, mennonite et quaker qui se développèrent à partir du calvinisme) deviendra l'objet principal de son investigation (83). Il met au jour des incidences aussi bien économiques que politiques de certaines convictions religieuses dont ces sectes sont porteuses. Mais celles-ci ne sont plus formulées à partir du dogme de la prédestination. En signalant déjà dans le contexte de la première stratégie que la doctrine et la pratique de la Cène ont des retombées politiques et sociales, Weber fournissait par là même une indication sur ce qui allait ultérieurement retenir son attention : l'organisation (Verfassung) sectaire et sa vie interne (84). L'appartenance à la communauté religieuse, la question de la nouvelle naissance et de l'engagement personnel prennent ici de l'importance. La discipline de la communauté religieuse n'induit pas des effets nécessairement identiques au niveau de la conduite de vie que ceux qui dérivent de «l'appropriation subjective » par l'individu de la religiosité ascétique, c'est-à-dire de la doctrine de la prédestination $(85)$.

La piété calviniste, en raison du dogme de la prédestination, exclut en principe que l'État soutienne la religion. Néanmoins, en mettant l'accent sur la gloire de Dieu à laquelle doit participer le chrétien, l'instrumentalisation de l'Etat peut

(81) ÉS, pp. 578-579 (trad. modifiée) ; ÉP, pp. 159-160 et note p. 214. Voir aussi Jean Martin OuÉDRAOGO, «Esquisse d'une sociologie de la Bible chez Max Weber », op. cit., pp. 417 sq. La nouveauté de l'apport de Luther réside bien dans cette évaluation positive du travail, qui donnera corps dans la culture protestante à l'idée de «vocation ». L'amour du prochain est reformulé par le puritanisme - à la différence du luthéranisme - dans les mêmes termes que ceux qui servent à expliciter le travail social du chrétien dans le monde.

(82) ÉP, pp. 111-112. Voir également SR., pp. 349-350.

(83) Ibid., pp. $150 \mathrm{sq}$. La seconde version du texte sur "Églises et sectes" initialement publiée dans le Frankfurter Zeitung, porte le titre «Églises et sectes en Amérique du Nord. Une esquisse des Églises et de la politique sociale », parue ensuite dans Christliche Welt, 20, 1906, 24. La troisième version du texte porte un tout autre titre (!) : «Les sectes protestantes et l'esprit du capitalisme » dans ÉP, pp. 255293. Voir la note introductive dans ibid., p. 256.

(84) Ibid., pp. 104 et 108 (ajout de 1920).

(85) Ibid., pp. 161-162. 
s'avérer nécessaire pour écraser l'hérésie. En revanche, la perspective sectaire résidera, du point de vue psychologique, dans le rejet du dogme de la prédestination et dans le fait de penser sous une même question la nouvelle naissance et l'appartenance à l'organisation religieuse. De cette manière, le problème de l'élection concerne aussi bien le participant ordinaire à la Cène (le laïc) que le prédicateur. À propos de ce dernier, le savoir théologique n'est plus une garantie pour être accepté comme membre de la communauté qui ne doit dorénavant réunir que des élus confirmés. La distinction prédicateur/laïc disparaît. L'Église, de leur point de vue, ne doit avoir en son sein que des régénérés. Du même coup, toute intervention externe, d'ordre politique notamment, dans la vie de la communauté doit être proscrite. Pour des régénérés, ce serait une offense à la gloire de Dieu que de participer à la Cène avec un réprouvé. Le "volontarisme" des groupes sectaires et le principe de la séparation entre Église et État (revendiqué très tôt par la tendance "calviniste baptiste" de l'armée de Cromwell) en furent les conséquences. Les sectes d'origine anabaptiste, dès leur origine, se sont illustrées par la défense de ce principe (86).

L'abandon de l'universalisme religieux manifesté dans l'orientation des groupes sectaires correspond à un choix théologique en matière d'organisation ecclésiale. L'option pour la forme associative comme mode d'organisation - à la différence du type institutionnel d'organisation - va de pair avec la revendication de la liberté de conscience et de la séparation des Églises de l'État. Ni "l’Église catholique", ni "l’Église luthérienne ancienne", ni "le calvinisme", ni non plus "la vieille Eglise baptiste", en raison de leur propension universaliste, ne peuvent être les porte-drapeaux de cette revendication, note Weber. L'engagement des Quakers, en particulier en faveur de la liberté de conscience, poursuit-il, ne se limite pas à la recherche d'une garantie juridique de leur propre existence dans la société ; il signifie une option en faveur du droit de tout croyant de pouvoir exercer librement sa religion. C'est ainsi, selon Weber, que ce droit à la liberté de conscience d'abord relatif à l'action religieuse s'étendra par la suite aux autres domaines de l'action sociale : la presse, la vie associative, etc., mais aussi à la liberté de conscience de la femme, c'est-à-dire, sa libération du pouvoir patriarcal (87). À l'origine de cette valeur moderne, se trouve donc une conception particulière du salut qui récuse toute pertinence quelconque aussi bien de l'autorité religieuse en cette matière, que de l'État. Quant au rejet de l'idolâtrie de la créature, c'est le propre de toute religiosité ascétique, toujours dans sa pure forme, ennemi de l'autorité (88).

C'est en Amérique que Weber voit se manifester le plus clairement les incidences politico-sociales du protestantisme sectaire. Il les perçoit dans le fonctionnement de la démocratie, l'ancrage constitutionnel des Droits de l'homme, la culture politique anti-autoritaire (ce qu'il appelle aussi l'absence de respect ("Respektlosigkeit”) des Américains), et la sécularisation de la société civile. Il

(86) Ibid., p. 131 note 1 , p. 150 note 4, p. 153 note 1 , p. 158.

(87) Voir ici, ÉP, p. 166, note 111 ; ÉP, note 22 pp. 210-212; SR, pp. 326 sq. Idem, Gesammelte Aufsätze zur Soziologie und Sozialpolitik, op. cit., pp. 431 sq. Il faut se reporter en particulier à l'esquisse de la sociologie de la presse, des associations, faite à l'occasion des premières journées de l'Association allemande de sociologie tenues à Francfort en 1910 pour avoir une idée de l'étendue du champ de recherche: les différentes sortes de domination dans la vie sociale moderne, la presse, l'opinion publique, etc.

(88) ÉP, pp. 191-92. 
impute l'émergence de ces dimensions de la vie moderne à l'influence du mode de fonctionnement interne des sectes, à l'abandon de tout universalisme de nature religieuse dont elles firent preuve et, enfin, au rejet de l'idolâtrie de la créature.

Ce qui intéressa Weber dans la démocratie en Amérique, c'est le processus de sélection (Auslese) des individus nécessaires à son fonctionnement. Cette forme d'organisation sociale rompt avec le principe général sur lequel repose la société d'ordres où l'origine familiale ou sociale détermine principalement l'accès de l'individu aux différents types de pouvoir dans la vie sociale. Ce que Weber désigne par "ordonnancement élastique" et par "individualisme" renvoie aux conditions générales de la mobilité sociale que manifeste le fonctionnement de cette démocratie (89). Cette mobilité sociale a été rendue possible par le rôle social que les sectes protestantes ont joué dans ce pays. Ce rôle social des sectes a été aussi renforcé par celui des associations de toutes sortes qui se sont organisées dans cette République américaine en suivant le modèle d'organisation sectaire. Ainsi, en raison de son mode de fonctionnement démocratique (pour les sectes, importance du principe du sacerdoce universel, du rôle des laïcs), ce terreau associatif a, dans son ensemble, entretenu "un rapport d'affinité" avec la démocratie (90).

Le projet de Weber est resté inachevé. Aucun texte n'est venu éclairer l'expérience historique anglaise, ni d'ailleurs, le lien entre puritanisme et politique de puissance. Mais l'inachèvement d'un projet ne signifie pas nécessairement sa remise en cause : ni du motif, ni de l'intention qui en sont à la base, ni non plus de son objet. Tout au moins invite-t-il à s'interroger sur les obstacles intrinsèques, éventuels qui ont pu freiner son plein déploiement. Quelques observations à ce sujet : notons au passage que le «tournant» qu'amorça Weber en 1915, avec ses études comparatives sur l'éthique économique des religions mondiales, ne peut être interprété comme une déviation par rapport à ce projet. D'abord, parce qu'il n'a, à aucun moment, remis en question les objectifs qu'il poursuivait. Ensuite, parce que le souci du comparatisme était présent dès l'Éthique protestante (91). Ce procédé a seulement gagné en envergure à partir de cette date. Il continua enfin ses investigations sur la base des hypothèses de départ (92).

L'inachèvement, tiendrait-il aux difficultés liées à la démarche même de Weber? Les problèmes résultent, nous semble-t-il, du fait que, dans ces études, il a attribué au puritanisme le statut de variable indépendante et émis l'hypothèse d'un rapport unitaire de cette culture au monde. Par la première opération, il est conduit à la recherche du type de pratique et d'institutions politiques susceptibles d'être induites par le puritanisme. Ses analyses ne se situent pas au niveau global du développement politique d'une société précise: pas même de celui des États-Unis à propos duquel il est plus prolixe. Peu de références historiques sont invoquées au

(89) Idem, Soziologie, Weltgeschichtliche Analysen. Politik, Stuttgart, Alfred Kroner, 1964, p. 393 ; idem, Gesammelte Aufsätze zur Soziologie und Sozialpolitik, op. cit., p. 443. Max Weber, (comme Jellinek d'ailleurs) n'était pas naïf au point d'idéaliser la situation américaine. Qu'il ait cherché à voir de plus près, la condition des Noirs dans ce pays, par exemple, ressort dans le texte ci-après ; cf. Laurence A. SCAFF, "The "Cool Objectivity of Sociation" ", History of the Human Sciences, II, 1998, 2, pp. 61-82.

(90) $S R$, p. 324.

(91) Cf. Jean Martin Ouédraogo, «Esquisse d'une Sociologie de la Bible », op. cit.

(92) Idem. Voir également la note 3 du présent texte. 
sujet de l'Angleterre. On est donc passé de l'expérience historique qu'aurait dû révéler l'histoire politique de l'Angleterre à celle d'une culture religieuse. Ce déplacement n'est pas sans conséquences. Weber avait conscience des difficultés de sa démarche. Même en admettant que ce qu'il réalisa effectivement ne pouvait être tout au plus - du point de vue programmatique - qu'une première phase de recherche, on ne peut s'empêcher de penser que ses hypothèses ne sont pas étrangères à l'inachèvement de son projet.

En effet, traitant le puritanisme comme variable indépendante, le problème est le suivant : dans quelle mesure, l'analyse d'une culture religieuse, du point de vue de son rapport à l'État par exemple, peut-elle être conduite sur la base d'une mise entre parenthèses, a priori, des contingences historiques ou plus généralement de l'ancrage historique réel de cette culture ? D'autant plus que Weber admit, par exemple, que la différence entre calvinisme et luthéranisme a été "politiquement" conditionnée (93). Autrement dit, il existe une variable politique à prendre aussi en ligne de compte dans l'analyse. Quant à l'hypothèse du rapport unitaire au monde, le problème apparaît en ces termes sous la plume de Weber : «l'ascèse intramondaine, [...] peut pactiser avec l'ordre politique de la violence qu'elle apprécie comme moyen de transformation éthique et rationnel du monde et de maîtrise du péché. Seulement, l'accord est ici loin d'être aussi facile qu'avec des intérêts économiques d'acquisition. (94)"Car, concède-t-il, à la grande différence de l'acquisition économique privée, l'activité politique, quant à elle, exige le compromis, la ruse, l'usage de moyens scandaleux du point de vue éthique, de ses semblables par exemple, la relativisation de tout but et l'abandon de toute exigence éthique rigoriste (95). La sphère politique semble donc avoir ses exigences propres, rendant ainsi problématique non seulement l'hypothèse du rapport unitaire quasi normal du puritanisme à l'économie et au politique, mais aussi celle qu'il prétendit, en pleine controverse, avoir mis en lumière; à savoir, que l'on pouvait, en ce qui concerne cette culture religieuse, comprendre en quelque sorte son orientation politique à partir de son orientation économique.

Quoi qu'il en soit, que Weber ait manifesté une intention politique très claire en s'engageant dans ce qui deviendra sa contribution en sociologie des religions, ses réflexions politiques sur la Russie, contemporaines du début de cet engagement semblent bien l'indiquer.

Dans ces textes, Weber s'identifiait au libéraux russes et assumait leurs visées politiques. En y cherchant à clarifier les conditions culturelles et politiques nécessaires à la transformation de ce pays, il continuait de réfléchir, mais indirectement, sur les problèmes politiques qu'il avait formulés à propos de l'Allemagne (96).

Le régime politique russe sous Nicolas II, aux prises avec les événements révolutionnaires de 1905 , hormis sa référence à la tradition et la caution religieuse dont il bénéficiait, manquait de légitimité démocratique. Selon Weber, ce régime

(93) ÉP, p. 237 , note 83.

(94) ES, p. 598 ; (traduction modifiée).

(95) Ibid.

(96) Ces textes sont désormais disponibles, dans leur totalité, dans le volume édité par W.J. Mommsen et D. Dalmann. Cf. Max Weber, Zur Russischen Revolution von 1905. Schriften und Reden, 1905-1912, Band 1/10 der MWG, Hrsg. von Wolfgang J. Mommsen und in Zusammenarbeit mit Dittmar Dahlmann, Tübingen, J.C.B. Mohr (Paul Siebeck), 1989. pp. 86-279 ; 293-692. 
n'arrivait à se maintenir qu'en s'appuyant sur une bureaucratie aux pratiques policières et arbitraires dont les intrigues lui paraissaient sans limite. Comme perspective de sortie de la crise politique, Weber, reprenant à son compte les revendications des démocrates russes, formula explicitement quatre exigences à satisfaire : 1) La garantie des droits individuels;2) La constitution d'un État de droit ; 3) Une réforme sociale selon le modèle européen et enfin ; 4) La promulgation d'une réforme agraire. Implicitement, Weber inclura un cinquième point à ce programme en intégrant l'idée selon laquelle, la Russie étant une grande nation, devrait aspirer, pour cette raison, à une politique de grande puissance (97). Il faut noter que cette dernière exigence et les deux premières sont subsumées par Weber sous ce qu'il désigne par «combat pour la liberté » (Freiheitskampf) (98). Un tel programme était-il réalisable dans les conditions qui prévalaient en Russie ? se demanda Weber. En considérant les positions politiques des différentes classes et couches sociales et les effets idéologiques du développement du capitalisme, il vit avec pessimisme les chances d'une régénération politique de ce pays. Mais son diagnostic ne porta pas seulement sur ces paramètres. Il en vint également à envisager la situation du point de vue de la culture politico-religieuse. Nous découvrons qu'à ce propos, Weber essaie d'introduire des données provenant de sa sociologie des religions.

Il constate d'abord que l'éthique économique et politique « des sectes rationalistes russes » les plus significatives était fondamentalement différente de celle de "l'ascèse intramondaine » (99). Ensuite, il fait remarquer que "l'individualisme politique des droits de l'homme », dont l'avènement en Occident fut conditionné par des convictions religieuses, n'est plus remobilisable parce que le capitalisme a définitivement sapé la croyance en l'harmonie des intérêts. Weber se situe ici dans une perspective jellinekienne qu'il revendique d'ailleurs dans son texte (100). Dans la mesure où il entend aussi par combat pour la liberté, la politique de puissance, cet aspect du programme est également envisagé par rapport à la culture politicoreligieuse censée l'avoir conditionné. À ce niveau, le lecteur se rend compte que Weber était encore en possession d'une hypothèse non-étayée : "Le développement historique de la liberté moderne, écrit-il, a été tributaire de constellations particulières qui ne se répèteront plus. Énumérons-en les plus importantes : dans les armées de Cromwell, dans l'Assemblée constituante française, et aujourd'hui encore, dans notre vie économique dans sa totalité, souffle le vent des mers lointaines : - mais aucune parcelle de terre n'est désormais disponible: les grandes régions intérieures du continent nord-américain, d'une part, de la Russie, d'autre part, aux surfaces monotones, favorables au schématisme, sont celles sur lesquelles le centre de gravité de la population de la culture occidentale avance inexorablement, comme autrefois dans l'Antiquité tardive ; deuxièmement, la singularité de la

(97) Ibid, pp. 125 et 165 .

(98) Ibid., pp. 270 ; 677-678. L'emploi de la même notion de liberté dans les deux cas a conduit visiblement Mommsen à en conclure qu'il recouvre un seul sens. Cf. Wolfgang J. MommSEN, « Max Weber and the Regeneration of Russia », The Journal of Modern History, 69, mars 1997, pp. 2 sq. On notera aussi que si le développement politique de la France « disparaît » de la sociologie des religions en vertu de la formulation de son hypothèse générale, il réapparaît et reprend sa place dans la vision que Weber avait de la politique de puissance.

(99) MWG, 1/10, pp. 164-165, note 42.

(100) Ibid. 
structure économique et sociale de l'époque du "capitalisme primitif" en Europe de l'Ouest; et troisièmement, la conquête de la vie par la science, le "retour sur luimême" de l'esprit.[...] - Enfin, certaines conceptions de valeurs idéales issues de la singularité historique concrète d'un univers défini de pensée religieuse agissant en conjonction avec plusieurs constellations politiques tout aussi bien singulières et leurs présuppositions matérielles, qui imprégnèrent ensemble la particularité "éthique" et les "valeurs culturelles" de l'homme moderne. (101)"

Autrement dit, qu'il s'agisse de l'inscription institutionnelle des droits de l'Homme, identifiée à l'accès de la bourgeoisie au pouvoir ou de la politique de puissance, la Russie de 1905 se trouvait dans une situation également défavorable du point de vue de la culture politico-religieuse. Si ces textes politiques sur la Russie peuvent être invoqués pour justifier l'intention politique qui sous-tend la sociologie des religions de Weber, c'est qu'ils manifestent, mis en parallèle avec l'éthique protestante notamment, une synchronie au regard du développement intellectuel de Max Weber.

\section{Conclusion}

Bien que le projet sociologique de Max Weber soit demeuré inachevé, nous disposons néanmoins de matériaux suffisants pour acquérir une vue sur la tâche qu'il se fixa dans ses recherches.

Nous nous sommes référé au politique comme catégorie d'analyse dans le titre donné à ce texte, sans pour autant nous en expliquer. Nous n'avions pas non plus envisagé de le faire parce que, au regard des questions que nous avions à traiter dans ce texte, la nécessité d'une telle explication ne s'imposait pas. Par cette référence, nous tenions cependant à marquer, d'entrée de jeu, nos distances par rapport à l'histoire de la réception qui s'est globalement focalisée, au niveau thématique, sur le rapport entre religion et économie chez Weber. L'alternative que nous proposons dans ce texte est la suivante : la réflexion qu'effectua Weber sur religion et économie, constituait un détour sur un itinéraire sensé l'aider à penser le rapport entre religion et politique. Du point de vue de l'historiographie du puritanisme, il introduit une nouvelle perspective de recherche.

$\mathrm{Au}$ point de départ, une question: qu'est-ce qui a pu permettre aussi bien l'accès de la bourgeoisie anglaise au pouvoir politique, que la transformation de l'État anglais en Etat impérial. Weber formule une hypothèse principale qui le conduit vers l'examen du rôle du puritanisme dans l'histoire de ce pays. Par rapport à cette tâche, il resta à mi-chemin dans ses recherches, bien qu'elle donna lieu à des travaux de grande ampleur qui le firent entrer dans l'histoire de la sociologie moderne.

Pour comprendre les stratégies de recherche suivies par Weber à propos du puritanisme, il faut tenir compte de deux hypothèses complémentaires à celle qu'il

(101) Ibid., pp. 270-271; (souligné par nous). 
énonça aussi à propos du rapport unitaire des religions au monde, à ses ordres et valeurs. Ce rapport se tisserait en fonction, d'une part des signes d'espérance que les religions donnent à voir à leurs adeptes confrontés à la misère du monde, et d'autre part, de leur organisation comme groupements.

La première hypothèse le mena à examiner la doctrine de la grâce véhiculée par le puritanisme (version calviniste). Dans cette culture, les signes que l'adepte est appelé à décrypter se situent dans la sphère professionnelle, économique, tout d'abord ; étant entendu qu'il n'existe de profession que dans une sphère du monde. Dans la mesure où, dans l'intérêt de l'adepte, cet appel comporte aussi un aspect performatif, l'exercice de la profession n'est pas pensé comme une simple manière d'être au monde, mais comme une vocation, c'est-à-dire, comme la seule manière d'attester la gloire de Dieu. Mais plus encore, l'exercice de la profession ainsi comprise comme vocation n'est pas non plus uniquement centrée sur l'ordre économique. Elle est pensée comme devant prévaloir également dans les autres sphères $\mathrm{du}$ monde, dans l'ordre politique notamment. Cette acception de la vocation associée à l'auto-perception du puritain comme instrument de Dieu, et à son rejet de l'idolâtrie de la créature, feront de lui le porte-drapeau d'une culture politique anti-autoritaire.

La seconde hypothèse le mena à se préoccuper de la manière dont les puritains (sectaires) sont organisés. Les sectes modifient la réponse antérieurement donnée à la question de l'élection. La leur, apparemment paradoxale, consiste à considérer comme élu, uniquement celui qui l'a été par la communauté des régénérés. Les signes de la nouvelle naissance sont ceux que reconnaît cette communauté. Ces puritains abandonnent ainsi les voies susceptibles de conduire à une approche universaliste du salut. Cette position et le rejet de l'idolâtrie de la créature qu'ils reprirent, firent d'eux des défenseurs de l'anti-autoritarisme aussi bien à l'intérieur qu'à l'extérieur de leur groupement. La modernisation politique de l'Amérique serait impensable si l'on faisait abstraction du rôle de ces sectes dans ce processus. Weber n'est pas arrivé à pousser plus loin ses réflexions qui apparaissent plutôt comme des considérations préliminaires à la tâche qu'il s'était assignée.

Deux opérations préalables nous ont été nécessaires pour appréhender cette tâche et l'exécution qu'elle a connue : un coup d'œil dans le dossier demeuré clos aux interrogations relatives à l'histoire de la sociologie des religions de Weber, et la remise à l'ordre du jour d'une question que l'on a cru avoir réglée. Il a fallu, en effet, revoir le dossier peu connu que représentent les études sur les ouvriers agricoles de l'Est de l'Elbe. C'est là que nous voyons progressivement émerger ce qui se cristallisa par la suite autour de sa sociologie des religions. C'est-à-dire, le lieu où nous trouvons les premières indications sur le motif et l'intention politiques qui commanderont ultérieurement ce qu'il entreprendra dans cette discipline. Dans ces écrits aussi, nous découvrons Weber confronté à la nécessité d'une réflexion sur l'expérience politique. Cette réflexion, nous la retrouvons déclinée et problématisée dans sa sociologie des religions. Il a fallu aussi remettre à l'ordre du jour la question de la filiation Jellinek/Weber. À cet égard, il faut se départir résolument de la thèse, dominante actuellement, mais qui ne résiste pas à l'analyse, selon laquelle Weber aurait orienté ses recherches sur les rapports entre religion et économie après avoir découvert Jellinek. Il les dirigea plutôt sur les rapports entre religion et politique après avoir retenu chez ce dernier une grande leçon à propos des processus politiques : à savoir, l'existence d'un lien entre transformation politique 
et culture politique. C'est en vertu de cette leçon qu'il décida de reprendre, à nouveaux frais, la question de l'expérience politique anglaise. En formulant l'hypothèse sur le rôle de la culture politique d'origine puritaine dans cette expérience, il emboîtait le pas, sur ce point encore, à Jellinek. Il privilégia ainsi à son tour cette hypothèse, après que Jellinek eût cherché à montrer sa valeur explicative à propos de l'avènement des idées et des institutions politiques modernes. Du point de vue heuristique, Weber prit néanmoins son autonomie : au niveau historiographique et programmatique. Cette autonomie n'est pas étrangère à l'intention politique originaire sous-jacente à son entreprise.

Afin de préciser cette intention de Weber, nous nous sommes proposé de considérer brièvement ses écrits sur la révolution russe de 1905. On y constate un réinvestissement de ses acquis sociologiques dans cette réflexion politique, tissant, pour ainsi dire, la grille de lecture dont il s'est servi pour jauger les chances politiques des libéraux russes, du point de vue du facteur politico-religieux.

Bien que Max Weber fut, par son action et ses prises de position politiques, un représentant du libéralisme de l'époque wilhelminienne, il n'en reste pas moins qu'il a été considéré, par les historiens des idées politiques, comme un libéral « étrange ». La raison ? L'absence chez ce penseur d'une réflexion fondamentale de type philosophique sur les valeurs politiques du libéralisme (102). Ce jugement, quoique fondé, semble néanmoins avoir fait l'impasse d'une prise en compte de sa sociologie des religions, du motif et de l'intention qui le guidèrent dans cette discipline.

Pour clore, revenons sur une question d'ordre historiographique déjà signalée : si l'on admet, comme nous avons tenté de le montrer, que Weber en est venu à traiter du rapport entre religion et économie, parce qu'il s'intéressait à celui qu'elle noue avec le politique; et si l'on admet en outre, que l'ensemble de sa démarche est suspendue notamment à l'hypothèse d'une relation unitaire des religions au monde (profession, État, famille), que peut-on dire aujourd'hui de cette manière d'écrire l'histoire religieuse ? Grâce à l'ouvrage sur l'Éthique protestante, et depuis les positions de Trœltsch, la méthodologie de Weber a pris ses marques dans l'histoire de la sociologie. Nous savons aussi que la réception de ce travail a été accompagnée par une controverse encore ininterrompue (103). Quelle que soit la valeur que l'on peut accorder à cette étude en vertu de son hypothèse principale (le rôle du puritanisme), il nous semble que son évaluation globale - de même que celle de ses écrits sur les autres cultures religieuses - ne peuvent faire l'économie d'un examen des implications de l'hypothèse sur le rapport des religions au monde.

\author{
Jean Martin OUÉDRAOGO \\ Fasse - Institut Catholique - Paris
}

(102) Cf. Wilhelm Hennis, Max Webers Fragestellung, op. cit., p. 199 ; David Beetham, Max Weber and The Theory of Modern Politics, Cambridge, Polity Press, 1985, p. 312 ; Robert EdEN, « Doing without Liberalism. Weber's Regime Politics », Political Theory, vol. 10, 1982, 3, p. 386.

(103) Cf. Ernst Troeltsch, «Die Kulturbedeutung des Calvinismus », in PE, p. 191. Sur une vue récente de la controverse, voir notamment Hartmut LEHMANN, "Max Weber "Protestantische Ethic" ", op. cit., pp. 9-29 ; Hartmut Lehmann, Guenther Roth, Weber's Protestant Ethic, op. cit. ; Raymond Boudon, "Protestantisme et capitalisme: bilan d'une controverse ", Commentaire, 22, hiver 1999-2000, 88, pp. 807-818. 


\section{Résumé}

Ce texte montre qu'en s'intéressant au rapport entre religion et économie, en fait, Max Weber effectuait un détour pour être à même de comprendre celui qui noue religion et politique. Dans le domaine des religions, il a inscrit ses réflexions dans le sillage des travaux de Georg Jellinek. Mais il se distingua de ce dernier et de ses prédécesseurs du point de vue de l'historiographie du puritanisme. Son entreprise fut fortement tributaire du motif et de l'intention politiques qui la commandèrent. Ainsi il conçut cette sociologie pour rendre compte des principes religieux qui permirent le double avènement de la bourgeoisie au pouvoir politique et de la politique de puissance en Angleterre. Ce projet n'a toutefois pas été mené à son terme.

\section{Abstract}

The present article demonstrates that Max Weber's interest in the relationship between religion and economy was a detour to enable him to understand its relationship with politics. In his religious studies, Weber conceived his ideas under the influence of Georg Jellinek. In regard to the historiography of puritanism, however, he differed with his predecessors and Jellinek's perspectives. His work owes a great deal to the political motive that lay behind it. In his sociology he sought to account for the religious principles that prompted the bourgeoisie to size political power and to power politics specialy in England. He did not however, complete the project.

\section{Resumen}

Este texto muestra que, interesándose en la relación entre religión y economía, de hecho Max Weber tomaba otro camino para acercarse a comprender los lazos que se establecen con la política. En el campo de las religiones, las reflexiones de Weber siguen los pasos de los trabajos de Georg Jellinek. Pero él se distinguió de este último $y$ de sus predecesores desde el punto de vista de la historiografía del puritanismo. Su empresa fue fuertemente tributaria del motivo y de la intención políticas que la guiaron. Él concibió esta sociología para dar cuenta de los principios religiosos que permitieron el doble advenimiento de la burguesía al poder político y de la política de poder en Inglaterra. Este proyecto no fue, sin embargo, terminado. 
